RADIO OBSERVATIONS RELATED TO STAR FORMATION

P. G. Mezger and Lindsey F. Smith

Max-Planck-Institut für Radioastronomie

Bonn, FRG

\title{
I. AN OVERVIEW
}

1) Some Basic Facts

Stars form out of the interstellar gas. Their observed masses range from 0.08 to $\sim 100 \mathrm{M}_{\odot}$. The birth rate of stars or the initial mass function (IMF) decreases rapidly with increasing stellar mass, approximately $\propto M^{-1.35}$ according to salpeter (1955). There is no reason to believe that protostars with masses less than $0.08 \mathrm{M}_{\odot}$ do not form and contract; however, they will never become main sequence (MS) stars. Stars with masses higher than $100 \mathrm{M}_{\odot}$ can exist as stable MS stars with lifetimes of $10^{6} \mathrm{yr}$; however, radiation pressure acting on dust grains prevents their formation (Larson and starrfield, 1971). For single protostars to contract, densities of $10^{3}$ to $10^{6}$ atoms $\mathrm{cm}^{-3}$ are required. The average density of the interstellar gas is about 1 atom $\mathrm{cm}^{-3}$. Therefore, the formation of dense and cool clouds in the interstellar gas must be the first step in the evolutionary track of stars forming out of the interstellar matter. About $60 \%$ of the total mass of interstellar gas in our Galaxy is contained in clouds with total densities of some $10^{2} \mathrm{~cm}^{-3}$ or more (Mezger and Smith, 1975); most of these clouds are located within galactic radii 4 to $13 \mathrm{Kpc}$ and in the inner part of the gaseous nuclear disk which surrounds the nucleus of the Galaxy. These are also the regions in our Galaxy where, at present, most stars are being formed.

While the IMF in the solar vicinity increases steadily up to the lower limit of stellar masses, the IMF of open clusters turns over at about $1 \mathrm{M}_{\odot}$ (van den Bergh, 1961. See also Fig. 11 and Sect. III.2).*)

\footnotetext{
*) Herbig (priv. comm.) informs us that the stellar mass at which this turnover occurs, varies from cluster to cluster. For the sake of simplicity we continue to define low-mass stars by $<1 \mathrm{M}_{\odot}$ and cluster stars (or higher mass stars) by $\gtrsim 1 \mathrm{M}_{\odot}$.
} 
This suggests that the IMF in the solar vicinity is the result of a superposition of two rather independent mechanisms of star formation: $\mathrm{All} O \mathrm{OB}$ stars and most of the higher mass stars $Z 1 \mathrm{M}_{\odot}$ are formed in open clusters and $O B$ associations. Low mass stars $<1 M_{\odot}$ on the other hand apparently can form as single stars out of relatively small and dense clouds (Herbig, 1970). We suggest that low-mass stars form rather continuously out of dense cloudlets or small condensations in extended tenuous molecular clouds; but that these two processes are not mutually exclusive: 0 stars may eventually form in extended clouds where, for a long time, only low-mass stars have formed. It has been suggested that T-Tauri associations represent the earlier stage and that they may eventually become OB associations (Mezger, 1971).

Where do stars form? In the following, we discriminate between main spiral arms and large cloud complexes in the interarm region. We also discriminate between giant HII regions, which require several early 0 stars, and small HII regions, which require only one $O$ or $B$ star, to account for their ionization. (Somewhat arbitrarily, the intrinsic flux density of a giant HII region is defined as $S_{5} D^{2} \geq 40 O$ (f.u.) (KpC) ${ }^{2}$, which is four times the intrinsic flux density of orion A.) Giant HII regions are known to define the main spiral structure in external galaxies. Georgelin and Georgelin (1976) used this fact to determine the spiral structure of our Galaxy on the basis of optical and radio observations of giant HII regions. They can locate all observed giant HII regions in four main spiral arms between galactic radii 4 and $13 \mathrm{kpc}$, each winding about $3 / 4$ of a revolution around the galactic center (Fig. 1). Note, however, that some of the largest HII regions are located in the central part of the nuclear disk, <200 pc from the nucleus of our Galaxy. A large number of small HII regions are located between the main spiral arms, and are often associated with dense clouds (CO-clouds). These small HII regions represent O star formation in the interarm region. By counting Lyman continuum (LyC) photons, Mezger and Smith (1975) estimate the present birth rate of 0 stars and find that about $70 \%$ are formed in main spiral arms and the rest are formed (in about equal fractions) in cloud complexes in the interarm region and in the inner part of the nuclear disk.

Formation of star clusters in main spiral arms and in cloud complexes in the interarm region can probably account for the formation of all OB stars (Roberts, 1957) and hence of most of the higher-mass stars $\gtrsim 1 \mathrm{M}_{\odot}$. Where do low-mass stars < $1 \mathrm{M}$ form? As will be shown in sect. II, radio observations can not help to answer this question. But one can easily show that less than $1 \%$ of the interstellar matter contained in clouds within galactic radii 4 and $13 \mathrm{kpc}$ is transformed into cluster stars while passing through a main spiral arm (see, e.g. Mezger, 1975). The main effect of compression of interstellar gas entering spiral arms appears to be the production of clouds, of which only a small fraction collapses further and forms $O$ star clusters and associations in the main spiral arm. Most of these clouds appear to travel as relatively stable objects into the interarm region and it may be within these clouds that low-mass stars form. 


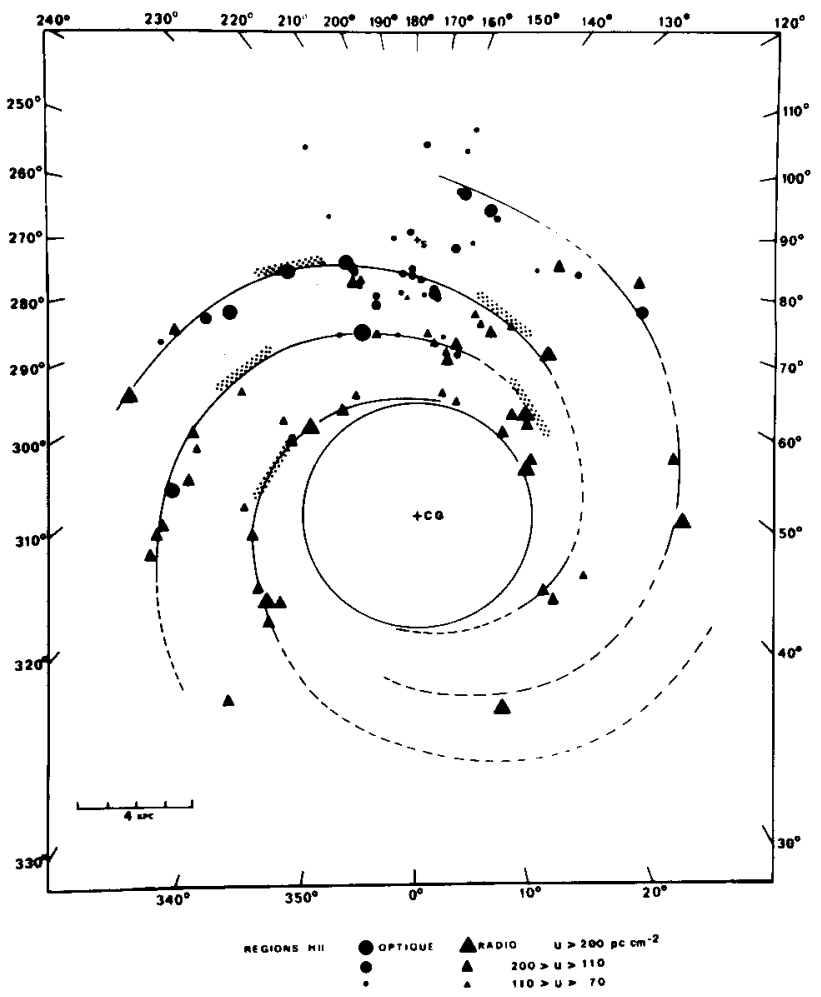

Figure 1: Main spiral arms in the Galaxy, determined from the distribution of giant HII regions by Georgelin and Georgelin (1976)

For stars $z 1 \mathrm{M}_{\odot}$, the units of star formation have been recognized as clusters or sub-group's which contain some 1 ooo $M_{\odot}$ of stars (Blaauw, 1964). There is no observational support for a picture where sub-groups form as the result of a central collapse of a spherical cloud. It rather appears that, in proto-clusters, physical conditions are such that single stars can form and that stars of $\sim_{1} M_{\odot}$ form first, followed by the formation of higher mass stars and ending with the formation of o stars (Iben and Talbot, 1966; Williams and Cremin, 1969).

What initiates star formation? As far as low-mass stars are concerned, we know nothing. As far as OB stars are concerned, we know that all giant HII regions, each of which contains several subgroups of 0 stars, are located in one of the four main spiral arms or in the central part of the nuclear disk; in cloud complexes in the interarm region apparently only one $O B$ cluster forms at a time although these clouds are nearly as massive as those associated with giant HII regions. This suggests that, in main spiral arms and in the central part of the nuclear disk, large-scale effects, causing a sudden compression of the interstellar gas, initiate collective star formation. In the interarm region, star formation appears to be dominated by more local effects, whose nature is not yet fully understood. Shock waves induced by supernovae may be a possible mechanism. Observations related to the formation of individual $O B$ stars, clusters and associations will be reviewed in sect. II. 
Stars form out of the interstellar gas and, in their final stages, return part of their mass to the surrounding interstellar matter, enriching it with elements heavier than $\mathrm{H}$. Therefore, the formation rate of massive stars is directly linked to the chemical composition of the interstellar gas. Today, in the solar vicinity, the mass fractions of elements are $\mathrm{X}\left({ }^{1} \mathrm{H}\right)=0.70, \mathrm{Y}\left({ }^{4} \mathrm{He}\right)=0.28$ and $\mathrm{Z}(\mathrm{A}>4)=0.02$. In other regions where more or less gas has been transformed into stars and subsequently returned to the interstellar matter, one expects metal abundances to be different from those observed for the solar vicinity. The connection between star formation rate and gas-to-total mass ratio on the one hand and between star formation rate and chemical composition on the other hand is investigated in sect. III.

\section{2) Radio and Sub-mm Observations Related to Star Formation}

Most stars form inside dense clouds where they can not be observed at optical wavelengths. Which kind of information related to the process of star formation can we hope to obtain from observations in the radio and sub-mm range? The main constituent of dense clouds is molecular $\mathrm{H}$, which is unobservable in the radio range. However other molecules such as $\mathrm{CO}, \mathrm{CS}, \mathrm{HCN}, \mathrm{NH}_{3}$ and $\mathrm{H}_{2} \mathrm{CO}$, which have transitions in the radio range, also exist in rather large numbers. Dickman (1975b) for example has shown that the ${ }^{13} \mathrm{CO}$ LTE column density can be directly related to the $\mathrm{H}_{2}$ column density for clouds with visual extinctions $1<A_{V}<10$. Regions of enhanced molecular emission can be the result of: higher optical depth (i.e. higher column density of molecules in a transparent cloud of constant temperature); higher gas temperature (for example in a cloud which is opaque in the CO transition); higher excitation, by collisions with $\mathrm{H}_{2}$ molecules (i.e. higher space density); or a combination of these effects. Combined observations of isotopic transitions (e.g. the ${ }^{12} \mathrm{CO}$ and ${ }^{13} \mathrm{CO} \mathrm{J}=1-\mathrm{O}$ transition) and of molecules with different dipole moments (e.g. the CO $\mathrm{J}=1-\mathrm{O}$ and the $\mathrm{CS} \mathrm{J}=3-2$ transitions) are needed to disentangle these effects. The general problem of how to interpret radio molecular lines is definitely outside the scope of this review.

Condensed objects, like protostars and MS stars, can be observed in the radio and sub-mm range if they interact with the surrounding interstellar matter. Examples of such interaction are: i) Heating of the surrounding gas, which results in an enhanced line emission; ii) Heating of the grains, which results in an enhanced IR and sub-mm emission; iii) Ionization of the gas, which results both in a free-free continuum and recombination line emission.

In the following, we discuss observations related to a wide variety of objects. We define:

a) Regions of enhanced molecular line emission or "dense molecular cloud". More or less extended regions in an usually much larger molecular cloud, where the $\mathrm{CO} \mathrm{J}=1-0$ transition has brightness temperatures $>10 \mathrm{~K}$, where the corresponding ${ }^{13} \mathrm{CO}$ transition is strong and where, usually, $\mathrm{mm}-1$ ines of molecules with higher dipole moment such as $\mathrm{CS}, \mathrm{HCN}, \mathrm{H}_{2} \mathrm{~S}$ and $\mathrm{H}_{2} \mathrm{CO}$ are observed in emission. 
b) IR stars: Unresolved (< 1") objects which emit primarly at IR wavelengths $<20 \mu$.

c) Far IR source: A more extended source spectrum usually can be roughly approximated by a black-body spectrum with temperatures $\hat{\gamma} 200 \mathrm{~K}$.

d) Giant HII region: Intrinsic flux density $S_{5} D^{2} \geq 400$ (f.u.) (Kpc) ${ }^{2}$.

e) Compact HII region: $10^{-3}<2 \mathrm{r}_{\mathrm{H}}+/ \mathrm{pc}<1 ; 10^{3}<\mathrm{n}_{\mathrm{e}} / \mathrm{cm}^{-3}<10^{6}$;

$10^{-3}<\mathrm{M}_{\mathrm{HII}} / \mathrm{M}_{\odot}<10$. An ionization bounded $(i b)$ compact HII region is embedded in a dense shell of neutral gas and dust. A density bounded (db) compact HII region is embedded in an extended low-density HII region.

f) Radio HII region: Has peak emission measure $\geq 10^{4} \mathrm{~cm}^{-6} \mathrm{pc}$.

g) $\mathrm{C}^{+}$region: $\mathrm{C}$ is ionized in a relatively large region (typcially

20.5 pc) while the associated $\mathrm{H}^{+}$region - if observable at all - is much smaller.

h) Protostellar shell: remnant of the protostellar cloud left behind after the central star has reached the MS. If an ib compact HII region has formed, the protostellar shell extends from the ionization front to the edge of the protostellar cloud.

i) Cocoon: Dust shell which absorbs radiation and reradiates it at IR wavelengths.

3) A Possible Evolutionary Sequence of $O$ and B Stars and their Protostellar Shells

The complexity of the subject warrants a reversal of the usual procedure; we introduce the review of observations (Sect. II) by outlining a working hypothesis for the evolutionary sequence of $O$ and $B$ stars. This sequence is based on radio and IR observations of the giant HII region W3 (reviewed by Mezger and wink, 1974) and on model calculations of dust-filled protostellar clouds and shells (Yorke and Krügel, 1976, and Yorke (in prep.)). Other papers to be mentioned in this context are those by Wynn-Williams et al (1972), Israel et al (1973), and Krügel and Mezger (1975) for observations and their interpretation; and by Davidson and Harwit (1967), Mathews (1969), Larson (1969a, b), Larson and Starrfield (1971) and Kahn (1974) for model calculations of various evolutionary stages. On the basis of this possible evolutionary sequence, we will (Sect. II) tentatively identify observed protostellar objects with evolutionary stages of protostars of different masses, and we will estimate the evolution times of these objects.

We take for granted the existence of a dense molecular cloud in which a dense blob of some $1000 \mathrm{M}_{\odot}$ of gas has condensed. In this blob, stars from $1 \mathrm{M}_{\odot}$ upward have formed, and have used up most of the gas. close to the center of the newly formed star cluster is a dense protostellar cloud of sufficient mass to form an $O$ or B star. Outside the star cluster (and in general between the condensations (blobs) of the large molecular cloud), the gas density is low (some $100 \mathrm{H}_{2}$ molecules $\mathrm{cm}^{-3}$ ), just enough to thermalize CO. Dynamical calculations by Yorke and Krügel (1976) start at the time when protostellar clouds of 150 and $50 \mathrm{M}$ respectively begin to collapse. More recent calculations by yorke (in prep.) refer to a protostellar cloud of $20 \mathrm{M}_{\odot}$. As a result of this collapse, MS stars of 36,17 and $12 \mathrm{M}_{\odot}$ respectively are formed which 
correspond to stars of spectral types $205-6$ to BO. These stars leave behind massive protostellar shells which contain fractions of 76 (66;40)\% of the mass of the protostellar cloud. At the time of the collapse, the radii of the protostellar clouds are of the order of $1 \mathrm{E} 18 \mathrm{~cm}$. The computations by Yorke and Krügel neglect rotation of the protostellar cloud and magnetic fields and treat the radiation transfer problem only roughly (diffusion approximation). Furthermore, some of the quantitative results may depend strongly on the assumed dust model (a mixture of graphite grains and graphite cores with ice mantles). Nevertheless, the predicted sequence of evolutionary stages appears to be realistic when compared to various IR and radio observations. These stages are (values referring to the $50 \mathrm{M}_{\odot}$ and $20 \mathrm{M}_{\odot}$ protostellar clouds are given in paranthesis):

a) $1.5 \mathrm{E} 5(3.2 \mathrm{E} 5 ; 4.7 \mathrm{E} 5)$ yr after the collapse has started and shortly after a star-like nucleus has formed at the center of the protostellar cloud, an inner dust cocoon of radius $5 \mathrm{E} 13(4.5 \mathrm{E} 13 ; 2.9 \mathrm{E} 13) \mathrm{cm}$ forms. Inside this cocoon, even refractory graphite grains evaporate. The inner cocoon would be an IR source of radius $1.6 \mathrm{E} 15(5.8 \mathrm{E} 14 ; 3.1 \mathrm{E} 14) \mathrm{cm}$ and effective temperature $400(540 ; 460) \mathrm{K}$. However, the collapsing protostellar cloud outside the inner cocoon is opaque in the IR. Therefore, an outside observer will only see an extended far IR source of much lower color temperature.

b) Radiation pressure from the inner dust cocoon acts on the dust component of the infalling material to which the neutral gas is coupled by friction. Finally, the mass flow is reversed and a second (outer) dust cocoon forms after $1.6 \mathrm{E} 5(3.6 \mathrm{E} 5 ; 6.1 \mathrm{E} 5)$ yr at a distance of $3 \mathrm{E} 17$ (1.E17; $4 \mathrm{E} 16) \mathrm{cm}$. In the subsequent evolution, both the inner and outer dust cocoon expand. An outside observer will see the inner cocoon as a hot IR source (color temperatures 400 to $1000 \mathrm{~K}$ ) and the outer cocoon as a far IR source (color temperature $\lesssim 200 \mathrm{~K}$ ). An HII region can only form inside the inner cocoon and will, in most cases, be unobservable at radio wavelengths.

c) After $1.9 \mathrm{E} 5(3.7 \mathrm{E} 5 ; 6.4 \mathrm{E} 5)$ yr the inner cocoon becomes optically thin to near IR and subsequently also to the UV and Lyc radiation. At this point, quantitative computations stop. During the $1 \mathrm{E} 3 \mathrm{yr}$ in which the star accretes its final mass of $36(17 ; 12) \mathrm{M}_{\odot^{\prime}}$ a very compact HII region forms inside the outer cocoon. An outside observer will see this very compact radio HII region together with the much extended far IR source formed by the outer cocoon. It is due to the formation of the outer cocoon by radiation pressure that nearly all far IR sources associated with compact HII region have the same spectral distribution, irrespective of the size of the compact HII region (Krügel and Mezger, 1975).

d) The compact HII region expands and soon the ionization front will meet the dust front in the outer cocoon. At this stage, an outside observer will see both a shell-like HII region and a far IR source of similar sizes.

e) In the following stages, an ib compact HII region will expand inside the protostellar shell. Its density will decrease while more and more of the neutral gas is ionized. An outside observer will see a compact HII region of constant flux density whose turnover frequency moves towards lower frequencies while the integrated IR luminosity decreases. 
f) With an expansion velocity of $\sim 10 \mathrm{~km} \mathrm{~s}^{-1}$, the ionization front will reach the edge of the protostellar shell in about $3 \mathrm{E} 4 \mathrm{yr}$. Starting from this point, a $d b$ compact HII region will evolve, whose total mass stays constant, but whose flux density decreases rapidly (see e.g. Mathews, 1969). It ceases to be a far IR source. The ionization front moves into the region of low gas density which surrounds the protostellar shell and thus forms an extended low-density HII region. The HII region ceases to be a "radio HII region" after the peak emission measure of the db compact HII region has decreased to values < $1 \mathrm{E} 4 \mathrm{~cm}^{-6} \mathrm{pc}$. Mezgex and Smith (1975) estimate an average life time of radio HII regions of $25 \mathrm{E} 5 \mathrm{yr}$.

Similar dynamical computations for smaller protostellar clouds out of which late $B$ stars may form have not yet been made. The evolutionary sequence for an early B star is similar to that described above but with the difference that the compact $\mathrm{H}^{+}$region is small but is accompanied by a rather conspicuous $\mathrm{C}^{+}$region. One may safely extrapolate that, for late $B$ stars with lower stellar mass, the mass fraction of the protostellar cloud left behind in the protostellar shell is lower too. This may prevent the formation of an outer cocoon and render the inner cocoon observable. If this picture is correct, early B stars should be visible as IR stars, far IR sources and $\mathrm{C}^{+}$regions; late $\mathrm{B}$ stars may be observable as IR stars only.

In comparing these evolutionary stages with observations, one must be aware that the most severe oversimplification in the model computations is probably the assumption of spherical symmetry of the protostellar shell, which determines the appearance of $i b$ compact HII regions and far IR sources. Nevertheless, the following evolutionary time scales for 0 stars should be realistic:

i) The O star reaches its zAMS stage after it has accreted the remnants of the inner cocoon and has thus attained its final mass. Only then can the ionization front move freely into the surrounding neutral gas. Thus, a modern theory of HII regions can still use the old fashioned approximation of "immediate turn-on" of the o stars. ii) During about one freefall time (for O stars, some $1 \mathrm{E} 5 \mathrm{yr}$ ) the collapsing protostellar cloud is unobservable. iii) Subsequently, for some $1 \mathrm{E} 4 \mathrm{yr}$, the protostar is observable as a far and near IR source with no radio counterpart. iv) After the $O$ star has reached its ZAMS stage, a compact HII region forms. Its lifetime as in ib HII region lasts typically some $1 \mathrm{E} 4 \mathrm{yr}$, provided the ionized gas does not reach pressure equilibrium with dense neutral gas. v) The stage of a radio HII region (i.e. the remnants of a $d b$ compact HII region with peak emission measure $1 \mathrm{E} 4 \mathrm{pc} \mathrm{cm}^{-6}$ embedded in an extended low-density HII region) lasts about 5E5 yr. The stage of an optically visible HII region (which requires emission measures $\lambda 3 \mathrm{E} 3 \mathrm{~cm}^{-6} \mathrm{pc}$ ) may last over the whole lifetime of an O star, depending on the extent and mass of the associated cloud.

This picture places optically visible HII regions relatively late in the evolutionary sequence of o stars. That is the reason that no sub-section on optical observations of HII regions is included in the following review. Mme Lortet-Zuckermann will report later on optical observations which she feels are relevant to the formation of $O$ stars and compact HII regions. 


\section{RADIO OBSERVATIONS RELATED TO THE FORMATION OF SINGLE STARS AND STAR CLUSTERS}

\section{Stars Less Massive than O Stars Associated with Dust Clouds}

Dense clouds of interstellar gas which are located sufficiently close to the sun are seen optically as dark clouds. They are often associated with T-Tauri stars which are considered to be pre MS stars in the mass range 0.5 to $2 \mathrm{M}_{\odot}$ (Herbig, 1962). These may be the regions in our Galaxy where preferentially low-mass stars form. The $\mathrm{CO}$ and ${ }^{13} \mathrm{CO}$ $\mathrm{J}=1-0$ emission from 34 dust clouds has been surveyed by Milman et al (1975); and from 68 dust clouds by Dickman (1975b). Typical values of the CO brightness temperature are $10 \mathrm{~K}$; since the co line is optically thick, this value is thought to be representative for the kinetic gas temperature in the clouds. Kinetic temperatures of this order can be maintained in dense gas clouds by: compressional heating during free-fall collapse; by ionization of carbon, if enough photons in the range 912 to $1101 \mathrm{~A}$ are available; and by ionization by relatively hard cosmic rays. The latter source of heating may be the most probable one for extended dust clouds, especially since ionization of dense interstellar clouds also appears to play an important role in interstellar gas phase chemistry involving ion molecules (see, e.g. Herbst and Klemperer, 1976). Milman (1975) finds enhanced molecular line emission only at the positions of NGC 2023, NGC 2068 and NGC 2024, which are well-known reflection or emission nebulae with exciting stars of spectral type early B to late o. From these observations and some calculations, Milman concludes that only stars with relatively strong UV emission (earlier than AO) can heat the surrounding gas by ionization of $\mathrm{C}$ and photo-ejection of electrons from dust grains, but that heating of the gas by collisions with dust grains (which are heated by absorption of stellar light) is a very inefficient heating process.

Herbig (1960) reasoned that massive protostars, whose pre MS contraction time is short, should still be closely associated with their protostellar gas cloud. He selected a number of nearby Ae and Be stars which, like T-Tauri stars, are closely associated with dust clouds. Loren et al (1973; for a review of further observations and their interpretation see also Loren, 1975) observed molecular line emission from dust clouds surrounding these $\mathrm{Ae}$ and $\mathrm{Be}$ stars and find enhanced $\mathrm{CO}$ emission in every case. CS emission from some of these regions indicates that they are both hotter and denser than the average cloud. While an early B star is not hot enough to form a strong $\mathrm{H}^{+}$region, it emits sufficient UV photons in the range $912 \leq \lambda / A^{\leq} 1101$ to form an extended $\mathrm{C}^{+}$ region. It can be observed through its $C$ radio recombination line emission which, at electron temperatures of $\sim 100 \mathrm{~K}$, is stronger than the free-free continuum. B stars are also luminous enough to heat the surrounding dust and thus to form a far IR source. This has been observationally demonstrated for the exciting B1.5 star of NGC 2023 (mentioned above as a source of enhanced molecular line emission) by Knapp et al (1975) and Emerson et al (1975). Pankonin and Walmsley (1976) analyzed its physical state. Both gas and dust temperature decreases from values of $\sim 100 \mathrm{~K}$ close to the star to about $10 \mathrm{~K}$ at the edge of the $\mathrm{C}^{+}$region 
$\left(r_{C^{+}} \sim 0.5 \mathrm{pc}\right.$ for an adopted total gas density of $\left.10^{4} \mathrm{~cm}^{-3}\right)$. But while $\mathrm{H}_{2}$ can exist, at least in the outer part of the $\mathrm{C}^{+}$region, $\mathrm{CO}$ would be dissociated in the $\mathrm{C}^{+}$region. If this calculation is correct, the $\mathrm{C}^{+}$region can not be the source of enhanced $\mathrm{CO}$ emission in the cloud L1630 as suggested by Milman.

\section{2) The Star Cluster in the Ophiuchus Dark Cloud}

A much investigated example of an optically opaque cloud where a star cluster is possibly forming deep inside is found in the ophiuchus dark cloud. This dust cloud, located at a distance of about $160 \mathrm{pc}$, extends over an area of several degrees. In an area of roughly $1.7 \times 1.2 \mathrm{pc}$, Vrba et al (1975) at $\lambda 2 \mu$ observed about 70 stars, most of which do not have an optical counterpart. The authors arrive at the following conclusions: The luminosity function derived for these stars is identical to that of other young clusters which can be observed optically; the observed IR stars are the brightest members of a star cluster embedded in a dust cloud; the extinction estimated for the brightest IR stars varies considerably and is much higher than the average extinction of the cloud, suggesting the presence of protostellar shells; the extinction law in the dense cloud is definitely different from the interstellar mean, suggesting the presence of larger size particles, as would be expected if icemantles form around refractory cores. Molecular line emission from the Ophiuchus dark cloud has been observed by various authors, the most com-

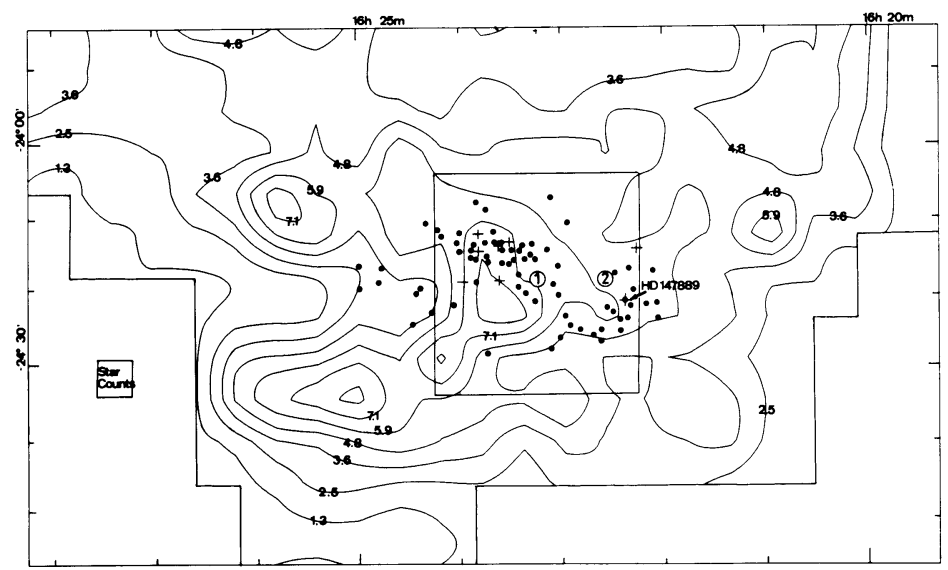

Figure 2: Map of visual extinction in the Ophiuchus dark cloud determined by star counts (Encrenaz et al, 1975). The curves are labelled by visual extinction, expressed in magnitudes. The positions of IR stars (Vrba et al, 1975) are indicated by dots. The corsses represent continuum sources which could be small compact HII regions. CO observations referr to the rectangle which includes most of the IR stars. 
p.lete investigation being that by Encrenaz et al (1975). Fig. 2 shows their map of visual extinction, superimposed on which are the IR stars observed by Vrba et al (1975). These IR stars cover only a small volume of the much more extended dust cloud. $\mathrm{CO}$ and ${ }^{13} \mathrm{CO}$ emission has been mapped within the rectangle shown in Fig. 2, which contains most of the IR stars. The total mass of gas contained within the rectangle (and thus associated with the main part of the IR star cluster) is estimated to be $\sim 2000 M_{\odot}$. Figure 3, from a forthcoming paper by Pankonin and Walmsley (in prep), shows the central part of the IR star cluster. G and VS numbers refer to the star lists by Grasdalen et al (1973) and Vrba et al (1975). C radio recombination lines have first been observed by Brown et al (1974). The vastly improved $\mathrm{C} 157 \alpha$ results by Pankonin and walmsley are shown in Figure 3; the heavy contours refer to the highest and lowest observed C157 $\alpha$ intensities. Brown and Zuckerman (1975) discovered a num-

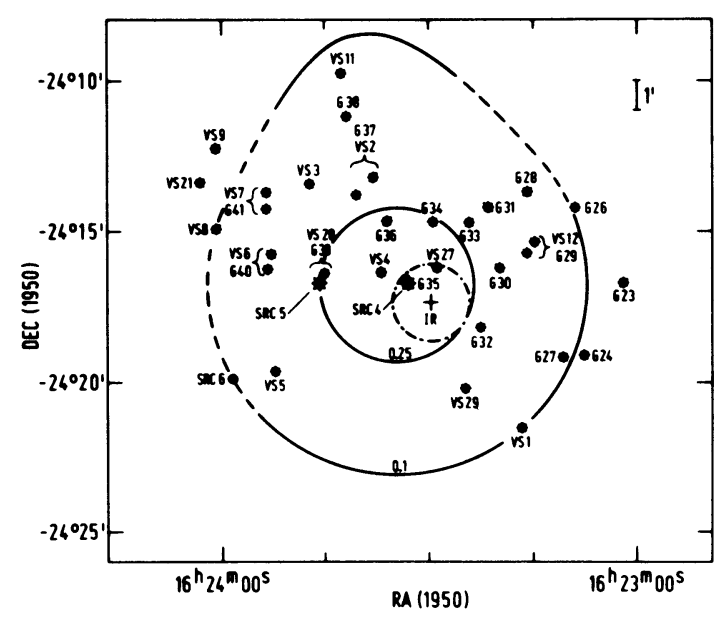

Figure 3: Central part of IR star cluster of Fig. 1 shown together with contour lines referring to the observed C157 line intensity (full lines) and far IR emission (dash-dotted line; Pankonin and Walmsley, in prep.). IR stars are labelled with VS and G numbers, weak continuum radio sources with SRC numbers. (For details, see text.)

ber of small continuum sources which they suggest to be weak compact HII regions; however, more recent $\lambda 21 \mathrm{~cm}$ observations with the Westerbork SRT (Encrenaz, priv. comm.) cast some doubt on the reality at least of the weaker sources. Continuum sources observed by Brown and Zuckerman are labelled with SRC in Fig. 3. Simon et al (1973) and later Fazio et al (1976) discovered far IR sources; the strongest far IR source from Fazio et al is indicated by its dash-dotted half power contour. The density of the molecular cloud peaks $\left(\mathrm{n}_{\mathrm{H}_{2}} \simeq 1 \mathrm{E} 5 \mathrm{~cm}^{-3}\right)$ close to the far IR source. All these observations appear to be consistent with a star cluster forming deep inside a molecular cloud. The most massive appear to be of spectral type early B, as witnessed by the presence of small compact $\mathrm{H}^{+} \mathrm{re}^{-}$ gions, an extended $\mathrm{C}^{+}$region and one or more far IR sources. Further, and consistent with the model calculations by Yorke and Krügel (Sect. I.3), the massive stars appear to be embedded in circumstellar shells. According to the ideas outlined in sect. I.1, one might expect that an 0 star will form next which would ionize and disperse the remaining gas so that the star cluster could become visible. While this picture fits in nicely 
with the evolutionary sequence of O star clusters outlined in Sect. I, one should be cautious not to overestimate the reliability of the various IR and radio observations on which the picture is based. We are rather certain that more accurate observations will necessitate modifications to the picture.

\section{3) O Stars Associated with Molecular Clouds}

The association of dense molecular clouds with HII regions is well known. The number $\mathrm{N}^{\prime}$ of Lyc-photons absorbed by the gas is proportional to the intrinsic radio flux density of the optically thin HII region. If $\mathrm{N}_{\mathrm{C}}^{\prime} \geq 2 \mathrm{E} 48$ Lyc-photons $\mathrm{s}^{-1}$, the ionizing star is of spectral type 09 or earlier. However, the reverse conclusion does not hold, since with increasing gas density more and more Lyc-photons get directly absorbed by dust grains so that, for densities $\chi 1 \mathrm{E} 5 \mathrm{~cm}^{-3}$, the HII region virtually ceases to exist (Mezger et al, 1974). Or, as outlined in sect. I.3, in the pre-ZAMS stage, all Lyc-photons get absorbed by the inner dust cocoon. Therefore, the total IR luminosity is usually a safer indicator of the spectral type of a young dust-embedded star and, if $\mathrm{L}_{I R} \gtrsim 10^{5} \mathrm{~L}_{\odot^{\prime}}$ the object is or will become an O star. The ratio $L_{I R} / N_{C}^{\prime} h \nu_{\alpha}$, ${ }_{1} . e$. the IR luminosity expressed in units of the energy availabte in the form of L $\alpha$ photons, is an indicator of the density of dust in the vicinity of the star. The following discussion relates to HII regions or far IR sources which are energized by $O$ stars according to the above mentioned criteria for $\mathrm{N}_{\mathrm{C}}^{\prime}$ and/or $\mathrm{L}_{I R}$. Liszt, in his unpublished thesis (1973; see also Penzias, 1975), presents a consistent set of data which will be referred to in this paragraph. Liszt mapped the $\mathrm{CO}$ and ${ }^{13} \mathrm{CO} \mathrm{J}=1-\mathrm{O}$ line emission from 25 clouds associated with HII regions. As far as we can judge, the maps relate to regions of enhanced CO emission which represent dense condensations of gas in a more extended but also more tenuous cloud. (See, as an example, the discussion of the orion region in the following paragraph). All investigated HII regions are associated with such dense clouds. Lower mass limits range from some $1 \mathrm{E} 2$ to $1 \mathrm{E} 3 \mathrm{M}_{\odot}$ for clouds associated with one O star cluster; and from some $1 \mathrm{E} 3$ to $1 \mathrm{E} 5{ }^{\circ} \mathrm{M}$ for clouds associated with giant HII regions. Liszt finds systematic differences between radial velocities of HII regions and molecular clouds: $\mathrm{V}_{\text {LSR }}(\mathrm{H} 109 \alpha)-\mathrm{v}_{\mathrm{LSR}}(\mathrm{CO})$ is negative for visible HII regions and positive for obscured HII regions. Liszt interprets this as an indication that, similar to the situation in Orion A (see following sub-section), HII regions are often ionization bounded in one direction (where the ionization front hits a dense neutral gas) but density bounded in other directions, where the ionized gas can freely expand. However, the validity of Liszt's statistics is questionable since many of his "obscured" HII regions are so remote that obscuration due to dust along the line of sight is more probable than obscuration by the molecular cloud itself.

Out of 60 Sharpless HII regions, Blair et al (1975) detected Co emission from associated molecular clouds in 30. Many of these optically visible HII regions relate to relatively late stages of their ionizing stars (Sect. I.3). The presence of $\mathrm{CO}$ clouds indicates that not all HII regions may be able to disperse the remnants of their parent clouds during their MS lifetimes. 
4) The Orion Region: Star Formation in an Interarm Cloud Complex

The Orion region is a well investigated example of an OB-star association, a compact HII region, and a dense molecular cloud, where the optically visible sub-groups of the association are in various evolutionary stages and where inside a dense condensation of gas, $O$ and $B$ stars form.

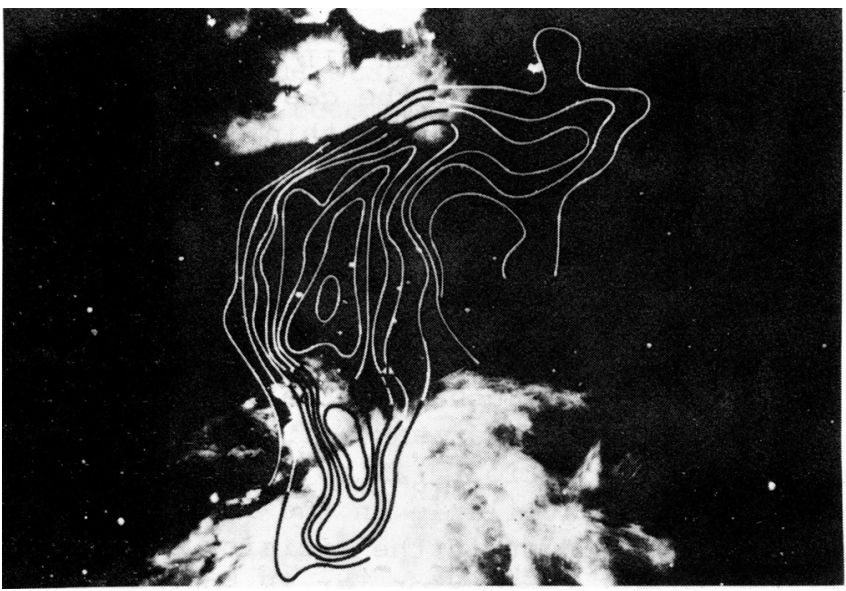

Figure $4:{ }^{13} \mathrm{CO}$ map of the dense orion molecular cloud, superimposed on a photograph showing Orion $\mathrm{A}(=\mathrm{M} 42+$ M43) south of the cloud and NGC $1973,5,7$ north of the cloud. (Kutner et al, 1976).

The Orion region is obviously not part of the main spiral structure of our Galaxy (see Fig. 1) and therefore maybe representative of o star formation in the interarm region. Figure 4 shows a ${ }^{13} \mathrm{CO}$ map of the Orion molecular cloud proper, a dense $\left(n_{\mathrm{H}_{2}} \sim 5 \mathrm{E} 4 \mathrm{~cm}^{-3}\right)$ cloud containing $\sim 2$ oOo $\mathrm{M}_{\odot}$ of gas (Kutner et al, 1976). The optically visible OB association extends $\sim 12^{\circ}$ from the molecular cloud to the north-west. It consists of four discernable sub-groups which differ in age by at least $1 \mathrm{E} 7 \mathrm{yr}$ (Blaauw, 1964). An extended region of weak CO emission extends from the molecular cloud over $\sim 5^{\circ}$ to the south-east (Kutner et al, in prep.). OB association and extended molecular cloud run approximately parallel to the galactic plane. The dense molecular cloud runs about perpendicular to the galactic plane; it is located at the interface between $O B$ association and extended cloud. At its southern end is located the compact HII region orion $A$ (which comprises M42 and M43) at the northern end is located the HII region NGC 1977. The southern and northern maxima in the ${ }^{13} \mathrm{CO}$ map (Fig; 4 ) are referred to as $\mathrm{OMC} 1$ and $\mathrm{OMC} 2$. OMC1 has a high density core $\left(\mathrm{n}_{\mathrm{H}_{2}}\right.$ $2 \mathrm{E} 6 \mathrm{~cm}^{-3}$ ) which comprises $200 \mathrm{M}$; OMC2 has a similar core, but less dense and massive. Orion $A, O M C 1$ and $O M C 2$ are far IR sources; the former two have luminosities of $1 \mathrm{E} 5 \mathrm{~L}_{\odot}$ which are typical for 0 -stars, while the IR Iuminosity of $\mathrm{OMC} 2$ is about a factor of a hundred less. The far IR source associated with Orion $A$ is extended and correlates well with the free-free emission. The far IR sources associated with OMC1 and OMC2 are much narrower ( $\left.\lesssim 1^{\prime}\right)$. The far IR source associacted with OMC1 is known as the Kleinmann-Low (KL) nebula, which comprises the Becklin-Neugebauer (BN) IR star (of color temperature $2600 \mathrm{~K}$ ) and a cluster of less luminous IR stars (Wynn-Williams and Becklin, 1974); similarly, a cluster of IR stars is located inside the far IR source associated with OMC2 (Gatley et al, 1974). 
$\lambda 1 \mathrm{~mm}$ emission from somewhat cooler dust grains is observed over a region 27' centered on OMC1 (Harvey et al, 1974) and at the peak of OMC2 (Hudson and Soifer, 1976). Various observational arguments suggest that orion A is ionization bounded on its rear side where the ionization front hits a dense molecular cloud, but is density bounded in other directions (see the review by Zuckerman, 1975, Lect. Notes Phys. 42, p360) *)

Are the Trapezium cluster and the IR star clusters separate subgroups? To answer this question let us tentatively identify: i) orion A as a $d b$ compact HII region. ii) The BN-KL IR complex as a late O star in a stage where both inner and outer dust cocoon are observable. This interpretation is supported by the recent detection of Brackett $\alpha$ emission from the vicinity of the BN star, indicating the presence of a very compact HII region $\left(\mathrm{n}_{\mathrm{e}} \succsim 3 \mathrm{E} 5 \mathrm{~cm}^{-3} ; \mathrm{r}_{\mathrm{H}}+\lesssim 5 \mathrm{E} 14 \mathrm{~cm}\right)$, such as one expects to form inside the inner dust cocoon (Grasdalen, 1976). iii) The IR stars in OMC2, we identify as a star cluster where stars up to late B have formed. We then conclude, on the basis of the evolutionary time scale estimated in Sect. I.3, that the evolutionary ages of these OB stars differ by probably less than $1 \mathrm{E} 5 \mathrm{yr}$; and that some $10^{5} \mathrm{yr}$ from now, the whole complex of stars associated with the dense orion molecular cloud may be seen as one sub-group of the orion association. This implies that $O$ and $B$ stars form within a short time interval in different parts of a sub-group; that therefore the ionization front of a $d b$ compact component is bound to interact with dense, neutral protostellar clouds of less evolved $O$ and $B$ stars; and that the actual units of star formation may be smaller than a sub-group (which, according to Blaauw, 1964, contains $\sim 2000 \mathrm{M}_{\odot}$ of stars) and may rather be represented by IR star clusters like those observed in OMC1 and OMC2.

In summary, the following picture emerges for the formation of $O B$ stars in the Orion complex; it may be typical for all large cloud complexes in the interarm region: Formation of o stars started at least $1 \mathrm{E} 7 \mathrm{yr}$ ago in the north-western corner of a molecular cloud which then extended over $20^{\circ}$. Subsequently, the formation of $O B$ stars proceeded in the direction of the Trapezium cluster, where O stars have formed recently. At present, OB stars close to the ZAMS are found in OMC1 and OMC2. This picture suggests that formation of 0 stars will proceed further, into the molecular cloud which extends to the south-east of the Trapezium. Star formation and subsequent dispersion of the remaining gas in the $O B$ association must have been rather efficient, since no $C O$ emission is detected there (Tucker, priv. comm. quoting observations made by G. Chin). What created the dense molecular clouds out of which $\mathrm{OB}$ stars and star clusters can form? We do not know. But one may visualize a compression wave moving across an extended molecular cloud with a speed of $\sim 100 \mathrm{pc} / 10^{7} \mathrm{yr} \simeq 10 \mathrm{~km} \mathrm{~s}^{-1}$, which creates the dense clouds and thus initiates star formation. Expanding HII regions and supernovae may play a role in driving this compression wave.

\footnotetext{
*) A symposium on HII regions and related topics, which summarizes radio and IR observations of HII regions up to 1974 was held in Mittelberg, Austria, January 1975. The proceedings of this symposium are published will be referred to as above. See ref. Lect. Notes Phys. 42.
} 
5) W3: Formation of O Stars in Main Spiral Arms

Giant HII regions require more than one early o star to account for their ionization. Giant HII regions define the main spiral arms in external galaxies. In our Galaxy, all observed giant HII regions can be accounted for as being located in four main spiral arms (see Fig. 1) and in the central part of the nuclear disk. Many giant HII regions have been observed in the radio range with an angular resolution of a few arc sec with synthesis radio telescopes. A full account of these observations up to early 1976 is given in Table 2 of Mezger and Wink (1976). With a few exceptions (all very remote giant HII regions such as W49 or G0.7-0.0), it is found that all giant HII regions can be resolved into compact components (sometimes superimposed on an extended background of low surface brightness) where each compact component could be ionized by one single early O star. This suggests that giant HII regions represent OB star associations which are composed of a number of subgroups similar to the Orion $O B$ association discussed in the previous paragraph. The main difference between $O B$ associations forming in cloud complexes in the interarm region and those forming in main (density wave) spiral arms being that, in main spiral arm OB associations, a large number of $O$ stars reach the MS nearly at the same time while, in interarm OB associations, there is a rather long time delay (of the order of the MS lifetime of O stars) between the formation of $O$ stars in adjacent subgroups.

The optically obscured HII region $\mathrm{w} 3$ is part of a giant complex of HII regions located in the Perseus arm at a distance of $\sim 3 \mathrm{Kpc}$ from the sun and $\sim 12 \mathrm{Kpc}$ from the galactic center. This complex also includes the optically visible HII regions IC1848, IC1805 and IC1795. A radio map of this region obtained at $\lambda 11 \mathrm{~cm}$ with an angular resolution of $\sim 11$ ' by Wendker and Altenhoff (in prep.) is shown in Fig. 5. IC1805, IC1795 and W3 appear to form one OB association where the age of the O stars (referred to ZAMS) decreases from < 5E5 yr at the eastern edge to zero in

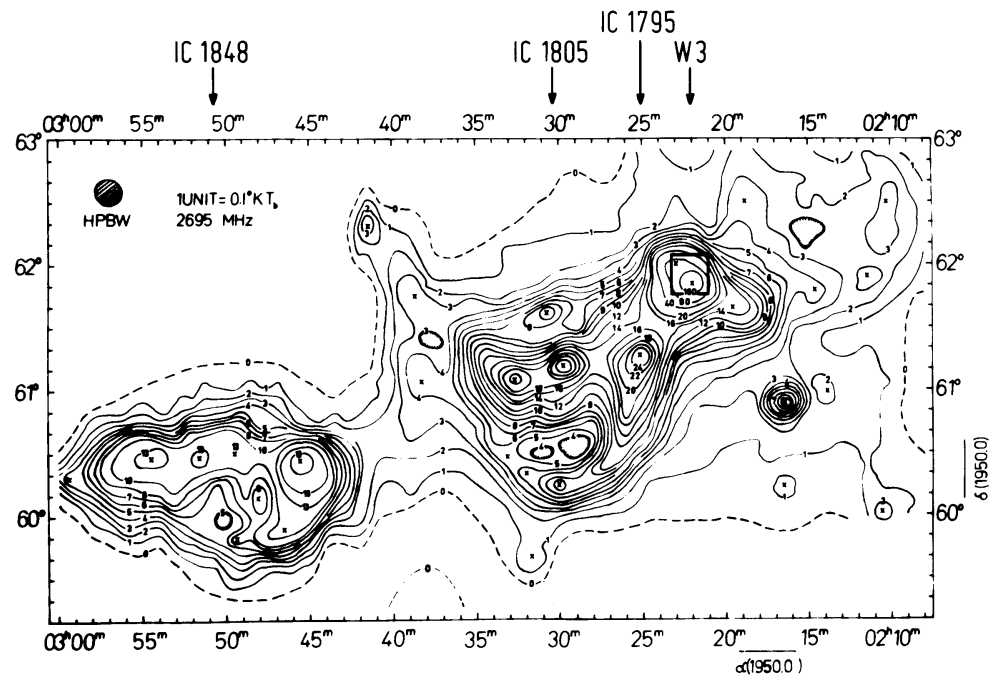

Figure 5: Contour map of IC1848, 1805,1795 and $w 3$ observed at $\lambda 11 \mathrm{~cm}$ with an angular resolution of $11^{\prime}$ (Wendker and Altenhoff, in prep.). The rectangle indicates the synthesized area shown in Fig. 5. 
the compact objects in $\mathrm{W} 3$. The intrinsic flux density is $\mathrm{S}_{2} \cdot \mathbf{7}$ (IC1805+ IC1795 + W3) $\times D(=3 \mathrm{Kpc})^{2}=3600$ (f.u.) (Kpc) ${ }^{2}$ (Wendker and Altenhoff, in prep.), i.e. 36 times the intrinsic flux density of the Orion Nebula. (This is a lower limit since IC1805 and IC1795 appear to be density bounded to a large extent.)

In the context of star formation in main spiral arms, w3 appears to be of most interest. Fig. 6, from Sullivan and Downes (1973), is an overlay on a Palomar red print of an aperture synthesis map of $w 3$ (angular resolution 25" $\times 28^{\prime \prime}$; the synthesized area is shown in Fig. 5 by a solid

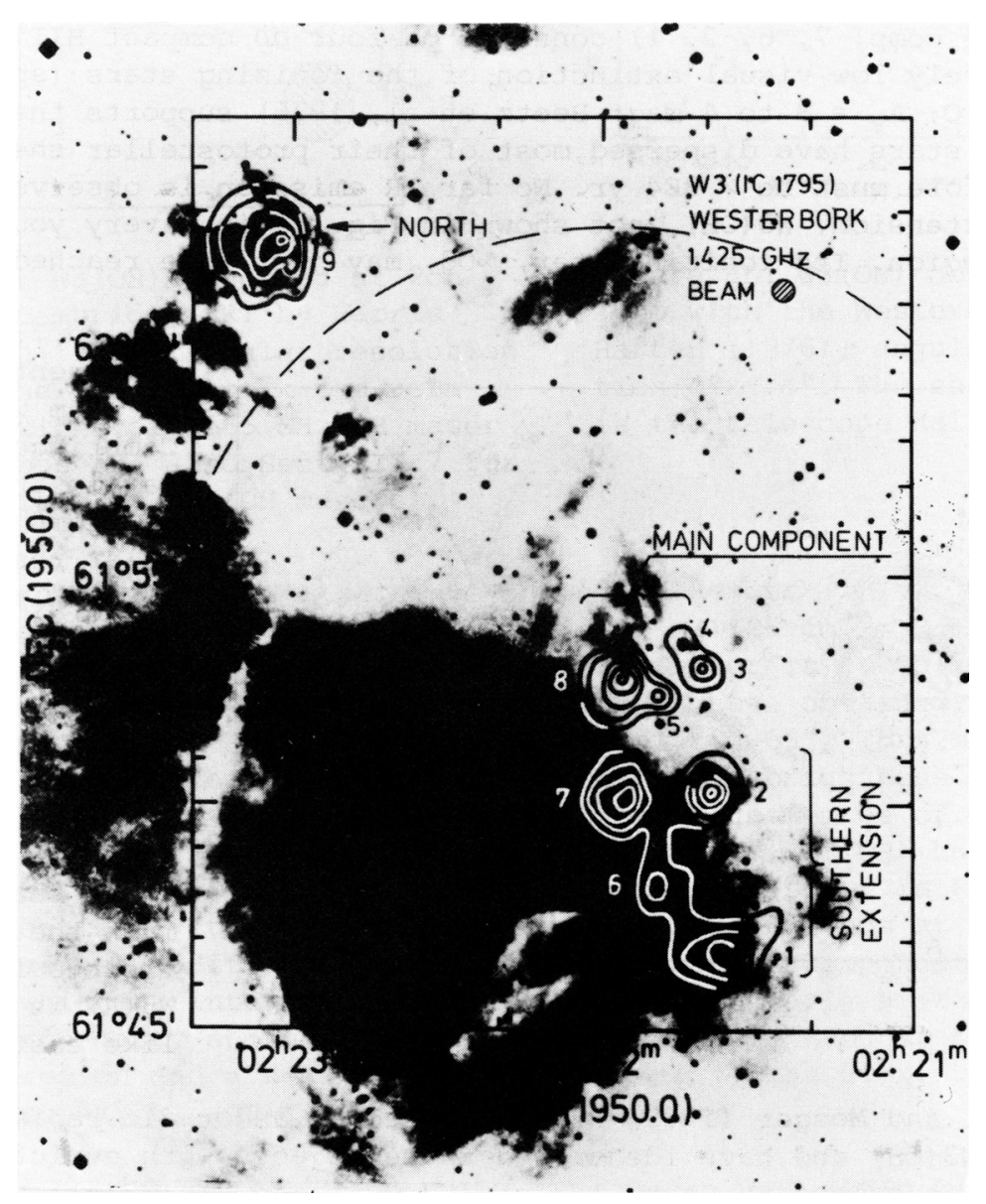

Figure 6: w3, Main component, Southern extension and Northern component observed at $\lambda 21 \mathrm{~cm}$ with the Westerbork SRT. Resolution 25"x28" (Sullivan and Downes, 1973). Compact HII regions $3,4,5$ and 8 are part of the Main component; $1,2,6$ and 7 are part of the Southern extension. $\mathrm{W} 3(\mathrm{OH})$ is located $17^{\prime}$ southeast of the Main component and outside the synthesized area. 
rectangle). About $17^{\prime}$ south-east of $W 3$, main component, and outside the synthesized map is $\mathrm{W} 3(\mathrm{OH})$, one of the most compact HII regions observed to date. For the following discussion, we refer to Mezger and Wink (1974; and 1975, Lect. Notes Phys. 42, p. 408). Only more recent results will be referenced explicitely.

In $W 3$, we recognize four regions of OB star formation. W3 (north) (= comp. 9 in Fig. 6), a db compact HII region, is ionized by a late O star of age (counted from ZAMS) $3 \mathrm{E} 4 \mathrm{Yr}$. W3 (main component) (= comp. 8, $5,4,3)$ contains seven $O B$ stars. Even the most evolved HII region, W3 (A), is still ionization bounded and sourrounded by its protostellar shell. Ages of ionizing stars therefore range from 0 to $\delta 3 \mathrm{E} 5 \mathrm{yr}$. W3 (southern extension) (= comp. $7,6,2,1)$ consists of four $d b$ compact HII regions. The relatively low visual extinction of the ionizing stars (spectral type $\mathrm{BO}$ or late $\mathrm{O} ; \mathrm{A}_{\mathrm{V}}=8$ to $4 \mathrm{mag}$; Beetz et al, 1976) supports the conclusion that these stars have dispersed most of their protostellar shells. Their ages therefore must be $>3 \mathrm{E} 4 \mathrm{yr}$. No far IR emission is observed from the southern extension. W3 $(\mathrm{OH})$, not shown in $\mathrm{Fig} .6$, is a very young ib compact HII region. Its ionizing star, w08, may just have reached the MS.

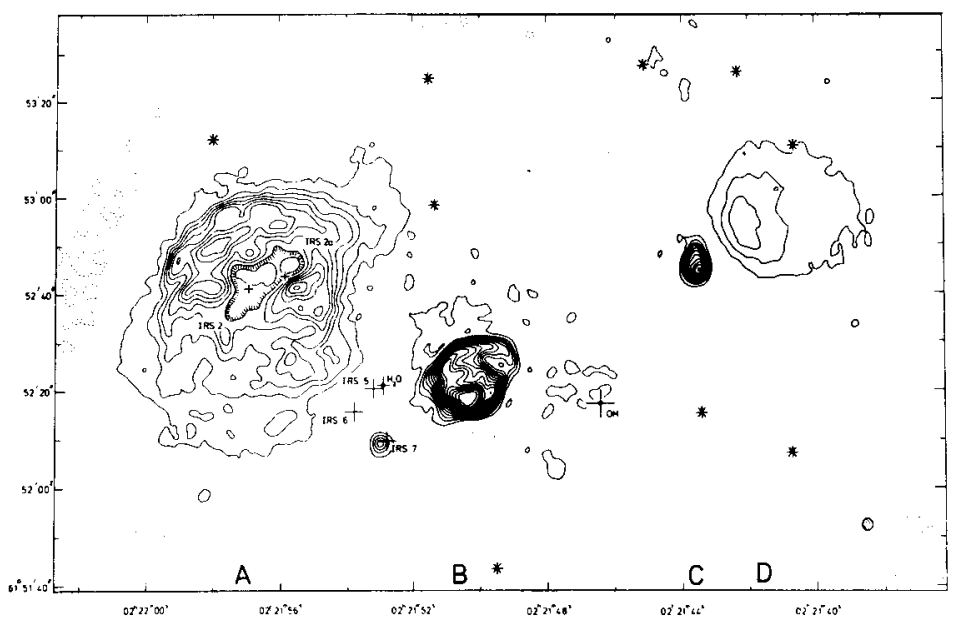

Figure 7: W3 (main component) observed at $\lambda 6 \mathrm{~cm}$ with the Cambridge SRT by Harris and WynnWilliams (1976); angular resolution $2 " \times 2.3 "$. Crosses mark the positions of IR stars and crosses with dots in the center mark positions of $\mathrm{OH}$ and $\mathrm{H}_{2} \mathrm{O}$ masers. The shaded ellipse is the half-power telescope beam. Asteriks mark the positions of visible field stars. W3 $(A)$ is the shelllike eastern component.

Krügel and Mezger (1975) have analysed IR and radio radiation from $\mathrm{W} 3(\mathrm{~A})$ and $\mathrm{W} 3(\mathrm{OH})$ and have identified these objects with evolutionary stages of O stars described in Sect. I.3.C) (W3(OH)) and d) (W3(A)) respectively. Harris and Wynn-Williams (1976) at $5 \mathrm{GHz}$ made an aperture synthesis map of W3 (main component) with an angular resolution of $2 " \times 2.3 "$ which is shown in Fig. 7. The main ionizing star of the shell-like eastern HII region W3(A) is IRS2; a somewhat less luminous star, IRS2a, has been detected in the near IR by Beetz et al (1974), at a projected distance of $2 \mathrm{E} 17 \mathrm{~cm}$ from IRS2. All HII regions shown in Figure 7 appear to be ionization bounded. 


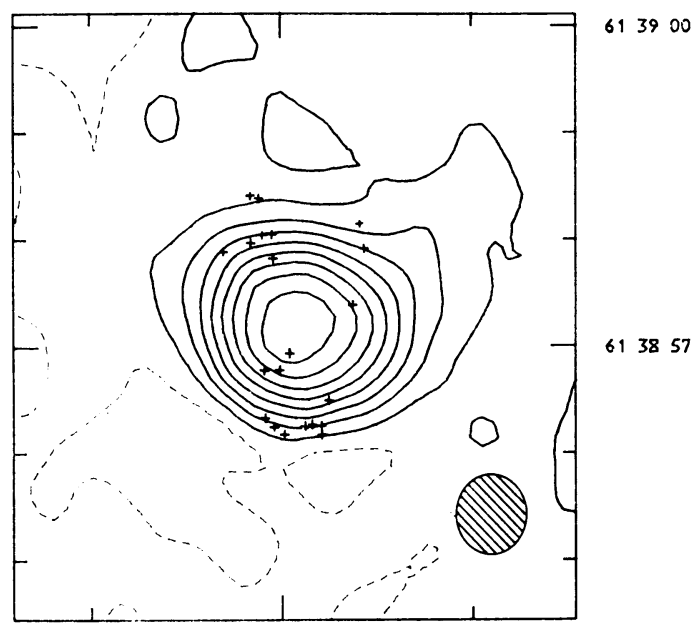

022316.75

022316.25

Figure 8a: W3 $(\mathrm{OH})$ observed at $2 \mathrm{~cm}$ with the Cambridge SRT by Harris and Scott (1976); angular resolution $0.65 "$. The crosses mark the relative positions of $1665 \mathrm{MHz}$ OH maser sources (but see also sect. II.7 for the positions of the $\mathrm{OH}$ masers).

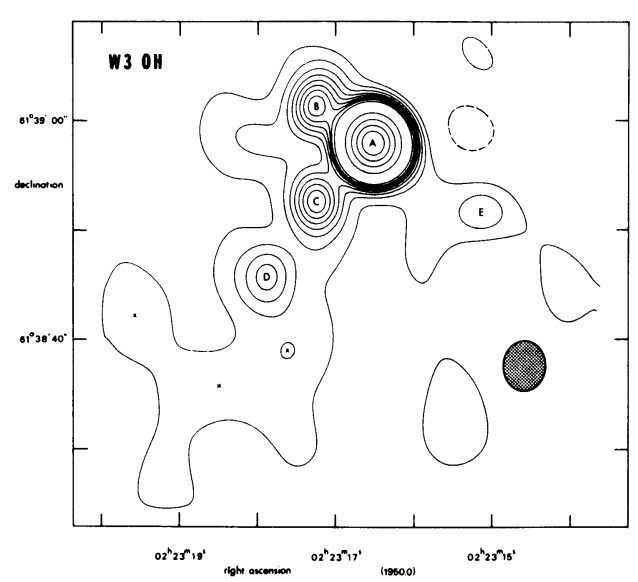

Figure 8b: W3 $(\mathrm{OH})$ observed at $\lambda 6 \mathrm{~cm}$ with the Westerbork SRT by Harten (1976); angular resolution 4" $x 4.4$ ". The shaded ellipse is the telescope half-power beam.

According to the analysis by Krügel and Mezger (1975), W3 (OH) con-

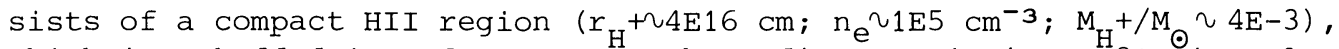
which is embedded in a dust cocoon whose diameter is 10 to 20 times larger. Recently, at $15 \mathrm{GHz}$, a map of $\mathrm{W} 3(\mathrm{OH})$ has been obtained by Harris and Scott (1976) with an angular resolution of 0.65". This map is shown in Fig. 8a. Although some structure is seen, the brightness distribution of the free-free emission still shows a very high degree of symmetry. At $5 \mathrm{GHz}$ and with an angular resolution of 4" $\mathrm{x} 4.4 "$ ", W3 (OH) has been mapped by Harten (1976). Besides the main component (shown in Fig. 8a) which is still optically thick at this frequency, Harten finds at least four, possibly seven, additional but much weaker compact components (shown in Fig. 8b) which he interprets as being ionized by early B stars. About 6' to the north-east, another, more evolved B star is seen as both a $d b$ compact HII region and a weak far IR source (Mezger and Wink, 1975, Lect. Notes Phys. 42, p. 408).

Liszt (1973)observed enhanced CO emission from dense clouds associated with $\mathrm{w} 3$ (main component) and $\mathrm{w} 3(\mathrm{OH})$ for which he estimates lower mass limits of $2 \mathrm{E} 3 \mathrm{M}_{\odot}$ and $9 \mathrm{E} 2 \mathrm{M}_{\odot}$, respectively. The $\mathrm{H} 56 \alpha$ line of $\mathrm{W} 3(\mathrm{OH})$ has been detected by Hughes and viner (1976). Its radial velocity $\mathrm{V}_{\text {LSR }}$ (H56 $\alpha$ ) is $-54 \mathrm{~km} \mathrm{~s}^{-1}$ and thus differs by $-7 \mathrm{~km} \mathrm{~s}^{-1}$ from the radial velocity of the CO cloud, $v_{\text {LSR }}(C O)=-47 \mathrm{~km} \mathrm{~s}^{-1}$, (Liszt, 1973). Not much is known about a more extended cloud of lower density which may encompass all four regions of $O B$ star association. Preliminary $C O$ observations appear to show that such a low-density cloud is present in those regions where the 
HII regions are obscured by dust (see Fig. 6), while CO emission disappears in regions of $\mathrm{H} \alpha$ emission (Thaddeus; Lada, priv. comm).

What can we learn about star formation in $O B$ associations in main spiral arms? A comparison with the orion region shows a number of correspondences but also some basic differences: The optically visible HII regions IC1848/1805/1795 can be compared to the subgroups in the Orion OB association, except that there appear to be many more subgroups whose ages probably differ by less than 5E5 yr. $d b$ compact HII regions such as W3 (north) and W3 (southern extension) can be compared to Orion A and NGC 1977. ib compact HII regions and IR sources without radio counter part in W3 (main component) and $\mathrm{W} 3(\mathrm{OH})$, which are embedded in dense molecular clouds, can be compared to OMC1 and OMC2. Again the number of OB stars close to or on the ZAMS is much larger in $\mathrm{W} 3$. There appears to exist a low density CO cloud which encompasses the four regions of OB star formation in W3. It would be highly interesting to know if a low density co cloud similar to that in the orion region extends to the west of W3, approximately parallel to the galactic plane. This comparison supports our introductory statement that giant HII regions represent OB associations in main spiral arms. The basic differences are the much larger number of subgroups and their much narrower age spread in the main spiral arm OB association. In the orion region, it appears that a compression wave proceeds from west to east, creating the physical conditions necessary for the formation of OB stars. In IC1848/1805/1795/W3 there appears to be a systematic decrease in evolutionary ages of the compact HII regions from east to west. However, whatever creates physical conditions for OB star formation in w3 (such as, for example, compression of the gas in the shock front of a density wave spiral arm) acts on a much global basis and appears to create more compact OB associations, i.e. the number of $O B$ stars per unit volume appears to be higher.

\section{6) Other HII Regions: A Review of Reviews}

Further reviews of observations related to the association of compact or giant HII regions, IR sources and molecular clouds are given in the proceedings of the Mittelberg symposium (1975, Lect. Notes Phys. 42) by D. Lemke for M17, S. Harris for DR 21, T. L. Wilson for W49, J. Bieging for W51 and a summary by A. H. M. Martin for Sgr B2. The association of ionized gas, IR sources and molecular clouds in the galactic center region have been reviewed by Mezger (1974) and Downes (1974). Other recent papers which are of interest in the context of star formation are those by Chaisson and Willson (1975) on M2O, by Lada et al (1976a) on M8 and Lada (1976) and Lada et al (1976b) on M.17, and by Israel (1976) on W58, a complex of extended and compact HII regions (which include $\mathrm{K} 3-50$ ), located in the Perseus arm at a distance of $\sim 9 \mathrm{Kpc}$ from the sun. The paper by Israel especially supports the picture that conditions for the formation of $O$ stars move from the edge of a cloud towards its center. The detection of an extended ( $85 \times 22 \mathrm{pc}$ ) molecular cloud of intermediate density $\left(\mathrm{n}_{\mathrm{H}} \simeq 5 \mathrm{E} 2 \mathrm{~cm}^{-3}\right)$ associated with M17 (Elmegreen and Lada, 1976) supports our conclusions in Sect. II, 4 and 5 that $O B$ associations form out of extended molecular clouds but the o stars will only form in those parts of the cloud where gas densities are one or two orders of magnitude higher than the average cloud density. It further confirms that parent 
clouds out of which OB associations form preferentially extend parallel to the galactic plane. Special attention should be given to the review paper by Habing (1975, Lect. Notes Phys., p. 156) on compact HII regions, in which he analyzes primarily westerbork synthesis observations of galactic HII regions. The true compact HII regions (which are centrally ionized) can be divided in those whose density decreases from the center outward; and those which show a shell-like structure. In the light of our discussion in sect. I.3, these two classes of compact HII regions are related to different evolutionary stages of protostellar shells. Besides these two genuine classes of compact HII regions, Habing finds another type of high-density HII region which is the result of an interaction of an ionization front with a dense cloud of interstellar matter. Orion A (sect. II.4) is the most famous example of such an interaction.

Radio observations of HII regions have recently been reviewed by Mezger and Wink (1976) with special attention being given to high resolution observations with single dish telescopes at high frequencies and with synthesis radio telescopes. It appears that the largest $i b$ compact HII regions have sizes $\simeq 2 \mathrm{E} 18 \mathrm{~cm}$ while $d b$ compact HII regions have sizes $\gtrsim 2 \mathrm{E} 18 \mathrm{~cm}$. Such a transition point from ib to $d b$ compact HII regions should occur when the ionization front reaches the edge of the protostellar cloud (Stage $f$ in the evolutionary sequence outlined in Sect. I. 3 ). A radius of $1 \mathrm{E} 18 \mathrm{~cm}$ for the protostellar cloud agrees well with the initial conditions for the collapsing protostellar cloud in the dynamical computations by Yorke and Krügel (1976). The observed masses of ionized gas in compact HII regions $\mathrm{M}_{\text {HII }} \lesssim 10 \mathrm{M}_{\odot}$ ' however, are smaller than the masses left over in the protostellar shells of their models. This discrepancy could be due to the fact that dense fragments of the protostellar shell remain neutral. In fact, there is mounting evidence of a high degree of clumping of the ionized gas. The size distribution of these hypothetical clumps has not yet been directly observed. But recent aperture synthesis observations of orion A by Gull and Martin (1975, Lect. Notes Phys. 42, p. 369) obtained with an angular resolution of 7.5 " $x$ 20" show that about $40 \%$ of the total flux density (but not of the mass of ionized gas) contained in the synthesized region is contained in structure of angular size $<1.3^{\prime}$ corresponding to linear sizes $<5 \mathrm{E} 17 \mathrm{~cm}$. Most of this fine structure appears to be unresolved, corresponding to linear sizes $\lesssim 8 \mathrm{E} 16 \mathrm{~cm}$. This result may have some bearing on the problem of fragmentation of a protocluster.

Not much information has been obtained relating to the process of star formation in the galactic center region, although a wealth of radio and IR observations have been published. The Sgr B2 molecular cloud, with a total mass of $3 \mathrm{E} 6 \mathrm{M}_{\odot}$, is probably the most massive cloud known in the Galaxy (Scoville et $\mathrm{al}, 1975$ ) and, in the giant HII regions GO.7-0.0 and GO.5-0.1 which are associated with this cloud, formation of O stars occurs at an extremely high rate. The nucleus of the Galaxy is surrounded by very massive compact HII region $\operatorname{Sgr} A$ West $\left(\mathrm{n}_{\mathrm{e}} \sim 1.4 \mathrm{E} 3 \mathrm{~cm}^{-3}, \mathrm{M}_{\mathrm{H}^{+}} \mathrm{M}_{\odot} \sim 6 \mathrm{E} 3\right.$ ). The nature of the source of ionization of Sgr A West is still not yet understood. 
7) Molecular Masers and Star Formation

In 1965 oH maser sources were discovered. In 1967 it became clear that class $I$ OH masers and compact HII regions are closely associated in space. Class I masers emit primarily in the central 1665 and $1667 \mathrm{MHz} \mathrm{OH}$ lines, which are highly polarized. In $1969 \mathrm{H}_{2} \mathrm{O}$ masers were detected, again in regions of active star formation. The $\mathrm{OH}$ and $\mathrm{H}_{2} \mathrm{O}$ masers occur as clusters of emitters, each of which exhibits a narrow line of width $\lesssim 1 \mathrm{~km} \mathrm{~s} \mathrm{~s}^{-1}$, apparent sizes of $1 \mathrm{E} 13$ to $1 \mathrm{E} 14 \mathrm{~cm}$ in size, and has brightness temperatures from $1 \mathrm{E} 13$ to $1 \mathrm{E} 15 \mathrm{~K}$. The cluster of emitters covers a region $1 \mathrm{E} 16$ to $1 \mathrm{E} 17 \mathrm{~cm}$ in size. The $\mathrm{H}_{2} \mathrm{O}$ masers change their intensities in time scales of months. Since the discovery of molecular masers, vast efforts by both observers and theorists have been made to explain this phenomenon. Nevertheless, the physical state of the gas in molecular maser sources is only known within rather wide upper and lower limits (say $1 \mathrm{E} 6$ to $1 \mathrm{E} 11 \mathrm{~cm}^{-3}$ for the density and 20 to $1000 \mathrm{~K}$ for the kinetic temperature). And the number of proposed pumping models has been reduced by elimination of the physically improbable models rather than by observational verification of predictions made. A review of molecular masers is outside the scope of this paper (but we would like to draw attention to the excellent review paper by strel'nitskii, 1975). In this section, we are concerned with one specific aspect: What can observations of molecular masers tell about star formation?

What is the relation between $\mathrm{OH} / \mathrm{H}_{2} \mathrm{O}$ masers and massive protostars or zAMS stars? The association of $\mathrm{OH}$ and (probably $d b$ ) compact HII region has been investigated by Habing et al (1974) who conclude that many, and probably all, class I OH masers occur near OB stars on, or close to, the ZAMS. These authors also suggest that the $\mathrm{OH}$ maser phenomena disappears when the $d b$ compact HII region has expanded to sizes $\gtrsim 2 E 17$, implying a lifetime of $\delta 1 \mathrm{E} 4 \mathrm{yr}$. At this distance, densities in the protstellar shells in Yorke and Krügel's models attain values $\mathrm{n}_{\mathrm{H}_{2}} \sim 1 \mathrm{E} 5 \mathrm{~cm}^{-3}$. If $\mathrm{OH}$ masers require gas densities $\gtrsim 1 \mathrm{E} 6 \mathrm{~cm}^{-3}$, their disapparence at this stage could be easily explained. According to Winnberg (priv. comm), to date 14 out of 18 class I OH masers (where both positions of maser sources and of compact HII regions have been measured with interferometers) coincide with compact HII regions with an rms uncertainty of $\sim 1 "$.

The association of $\mathrm{H}_{2} \mathrm{O}$ masers with $\mathrm{OH}$ masers and with other protostellar objects or ZAMS stars has been investigated by Lo (1974; see also the review by Burke, 1975, Lect. Notes Phys. 42, p. 188; and Lo et al, 1975a). He finds that $\mathrm{H}_{2} \mathrm{O}$ masers - like OH masers - are found in regions of active $\mathrm{OB}$ star formation; but that $\mathrm{H}_{2} \mathrm{O}$ masers - unlike $\mathrm{OH}$ masers are either not associated with compact HII regions or, if they are associated, their positions do not coincide. On the basis of this coincidence or lack of coincidence, together with the observational or circumstantial evidence for quite different dynamical time scales of $\mathrm{H}_{2} \mathrm{O}$ and $\mathrm{OH}$ masers (see above), LO suggests that $\mathrm{H}_{2} \mathrm{O}$ masers represent an earlier evolutionary stage of massive protostars which occurs prior to the formation of $d b$ compact HII regions. Recent simultaneous VLBI observations of $\mathrm{OH} / \mathrm{H}_{2} \mathrm{O}$ masers by Mader et al (1975) in $\mathrm{W} 3(\mathrm{OH})$ and $\mathrm{W} 49 \mathrm{~N}$ support this picture. Their result for $\mathrm{W} 3(\mathrm{OH})$ is shown in Fig. 8. The two clusters 
of $\mathrm{OH} / \mathrm{H}_{2} \mathrm{O}$ emitters are distinctly separated by a projected distance of $\sim 3.4 \mathrm{E} 17 \mathrm{~cm}$ (for $\mathrm{D}=3 \mathrm{KpC}$ ). Comparison with accurate interferometer positions of the $\mathrm{OH}$ masers (Welsh, priv. comm.) shows that the OH maser is associated with the ib compact HII region but that the cluster of oH emitters lies at its western edge (and not surrounding it as suggested in $\mathrm{Fig}$. 8a) while the $\mathrm{H}_{2} \mathrm{O}$ maser has no radio or IR counterpart.

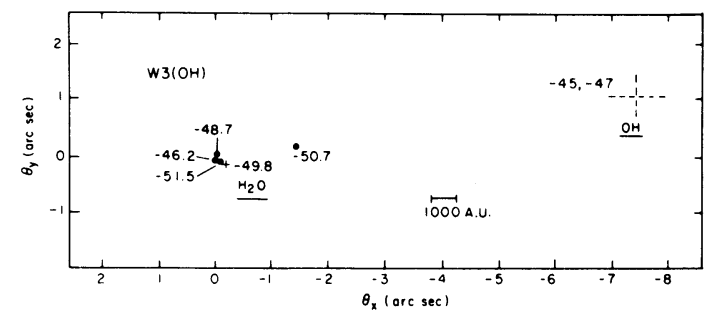

Figure 9: Relative positions of the $\mathrm{OH}$ and $\mathrm{H}_{2} \mathrm{O}$ masers in $\mathrm{W} 3(\mathrm{OH})$ derived by Mader et al (1975). The indicated $\mathrm{OH}$ position is a mean position for the blended features between -45 and $47 \mathrm{~km} \mathrm{~s}^{-1}$.

Burdjuzha et al (1975, Lect. Notes Phys. 42, p. 195) suggest a somewhat different (and, in the light of recent observations, less probable) model where, in ib compact $\mathrm{HII}$ regions, $\mathrm{H}_{2} \mathrm{O}$ masers are located in the transition zone HII-HI, while $\mathrm{OH}$ masers are located farther out in the protostellar shell between ionization front and preceeding shock front.

The pattern of circular polarization observed in $\mathrm{OH}$ masers can be interpreted as zeeman splitting; this yields magnetic fields of typically a few mG (Davies, 1974). More recent observations by Lo et al (1975b) yield $6 \mathrm{mG}$ for $\mathrm{W} 3(\mathrm{OH})$. Zeeman splitting of the $\lambda 21 \mathrm{~cm}$ hyperfine structure line suggests a general magnetic field of $2 \mu \mathrm{G}$ in clouds of density $\mathrm{n}_{\mathrm{H}} \sim 1 \mathrm{~cm}^{-3}$. If this field is frozen into the gas, its field strength must increase $\propto \mathrm{n}_{\mathrm{H}}^{\alpha}$ with $\alpha<2 / 3$ (see Mestel, this volume). Magnetic fields derived for other $\mathrm{OH}$ masers are similar. They are compatible with densities $\sim 1 \mathrm{E} 6 \mathrm{~cm}^{-3}$ estimated on the basis of realistic pumping models of $\mathrm{OH}$ masers.

A third class of masers detected in 1974 by Snyder and Buhl occurs in the rotational transitions $J=1 \rightarrow 0$, and $2 \rightarrow 1$ of the first vibrational state $(v=1)$ of the SiO molecule. Sio masers appear generally to be associated with late type (M3 - M9) Mira variables. No SiO masers are associated with $\mathrm{OH} / \mathrm{H}_{2} \mathrm{O} / \mathrm{IR}$ sources in, or close to, $\mathrm{HII}$ regions, with the only exception of an Sio maser observed in the direction of the KL nebula in the Orion region (Snyder and Buhl, 1975).

In summary: Both $\mathrm{H}_{2} \mathrm{O}$ and $\mathrm{OH}$ masers appear to occur in massive protostars and protostellar shells. $\mathrm{H}_{2} \mathrm{O}$ masers probably have gas densities of $\sim 1 \mathrm{E} 9 \mathrm{~cm}^{-3}$ and kinetic temperatures of $\sim 1 \mathrm{E} 3 \mathrm{~K}$; they occur before a compact HII region forms. In the evolutionary sequence outlined in sect. I. 3 , the $\mathrm{H}_{2} \mathrm{O}$ maser phenomenon would be compatible with the evolutionary stages a) and $\mathrm{b})$, with the emitting regions located close to the inner dust cocoon. Densities and temperatures in $\mathrm{OH}$ masers are probably considerably lower, say $\sim 1 \mathrm{E} 6 \mathrm{~cm}^{-3}$ and $\sim \mathrm{E} 2 \mathrm{~K}$; they occur when $\mathrm{db}$ compact $\mathrm{HII}$ regions have formed and would be compatible with stages d) and e), with the emitting regions being located just outside the ionization front. Although not much information related to the physical state of the gas can be deduced, $\mathrm{H}_{2} \mathrm{O}$ masers especially may be the earliest observational manifestation of a 
pre o star. Sio masers appear to be associated with late type stars in post MS evolutionary stages and thus are not of much interest in the context of star formation, but may be helpful to discriminate between pre MS and post MS objects.

\section{STAR FORMATION RATES IN THE GALAXY}

\section{1) As a Function of Density}

Radio observations of giant HII regions can be used to determine the rate of star formation (Mezger \& Smith, 1975, hereafter referred to as MS). Detection of giant HII regions is nearly complete for our half of the Galaxy, and the number of Lyman continuum photons needed for their ionization can be calculated. If the initial mass function (IMF) is constant over the Galaxy, the photon rate is proportional to the star formation rate. MS derived the rate as a function of distance from the Galactic center; the total value was $4 \mathrm{M}_{\odot}$ /year throughout the Galaxy derived from a total photon flux in giant ${ }^{\circ}$ HI regions of $4810^{51} \mathrm{sec}^{-1}$.

The star formation rate is, a priori, expected to be a function of many parameters: amount of gas available, metal abundance, strength of shock waves, and probably others. The parameter which has been singled out in the past for the most attention is the gas density, $\rho$. Schmidt (1959) favoured a dependence on $\rho^{2}$; however, none of the arguments he used at that time remain valid.

An apparently straight forward way to determine the exponent of such a law has been used by Emerson (1974) and by Madore et al (1974); earlier attempts are referenced by the latter. They plot HI column densities versus blue star counts or HII region counts (indicators of star formation). Both encountered difficulties. Emerson (whose results cover the region of greatest HI density in M31) noted that young and old OB associations and HII regions produce different results. Madore et al noted that the inner and outer regions of M33 give different results.

There are several aspects to be noted about the above experiments: 1. They do not allow for the presence of molecular $\mathrm{H}$, now known to account for most of the gas in our own Galaxy; 2. They use column densities rather than volume densities; 3. They use data from different regions of the galaxy.

For comparison with the work of Emerson and of Madore et al, we plot in Fig. $10 \log N_{C}$ versus $\log \left(M_{g a s}\right)$ for our own Galaxy from Smith, Biermann \& Mezger (in preparation, hereafter referred to as $\mathrm{SBM}$ ). $\mathrm{N}_{\mathrm{C}}$ is the number of Lyman continuum photons absorbed in giant HII regions, and includes a correction for absorption by dust inside the HII region. Mgas includes an estimate of the molecular $\mathrm{H}$ density from co observations (ranging from $80 \%$ of the gas at $5 \mathrm{kpc}$ from the center to $50 \%$ at $10 \mathrm{Kpc}$ ); somewhat better estimates can probably be made with more recent co studies (Gordon and Burton, 1976). Both quantities plotted are surface densities averaged over annuli in the galactic plane. The gas between 4 and 


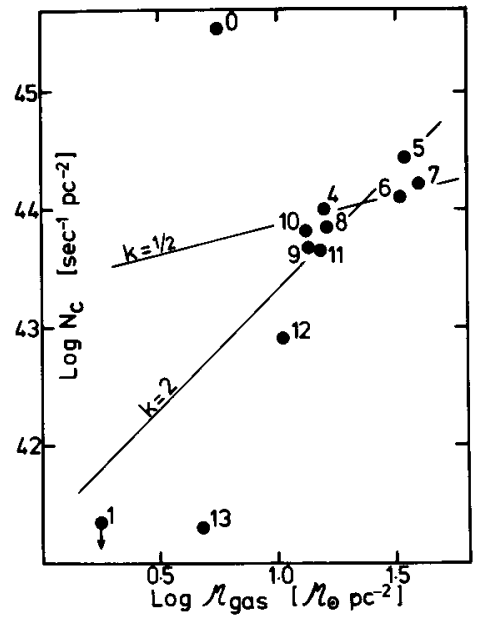

Figure 10: Plot of $\log \mathrm{N}_{\mathrm{C}}$ versus $\log$ Mas for annuli in the Galaxy. The points are labelled with the inner radius limit. The lines indicate slopes of $1 / 2$ and 2 .

$13 \mathrm{Kpc}$ is mostly $\mathrm{H}_{2}$, for which the scale height is $270 \mathrm{pc}$ out to $8 \mathrm{Kpc}$ and may then increase very slowly. Changes in mean density of the gas appear to reflect changes in numbers of clouds, not changes of the density or size of the clouds. Thus, we expect the total amount of gas available, rather than the mean volume density, to be the more physically relevant parameter.

The problem with this method of determining the slope is made reasonable clear by Fig. 10. Between 4 and $12 \mathrm{Kpc}$, the main spiral arms, the most gas and the most star formation occur; the observed points do not cover a sufficient range to define a slope. Within this area, a common law between star formation and density might be expected to apply because the process in all spiral arms is qualitatively similar. However, the metal abundance probably varies and the strengths of the shock fronts on the inner edges of the spiral arms (presumably a critical parameter (Woodward, 1976)) varies in strength with distance from the center. Thus the amount of gas is probably not the most important factor that changes from one annulus to another.

In the galactic center, the rate of star formation is probably controlled by different factors, as must also be the case in all regions $<4$ and $>12 \mathrm{Kpc}$ from the center, outside the region of main spiral structure. The galactic center point falls 2 orders of magnitude higher than is compatible with any simple relation; the other points fall far below. We conclude that there is little support for a common law representing. the star formation rate at different positions in the Galaxy.

\section{2) As a Function of Time}

We now apply a different approach. We look for the dependence of the star formation rate on time, and use the surface density of gas as a parameter. The surface density is probably a good parameter in as much as the amount of gas remaining in a given annulus (assumed isolated from all others) is probably monotonically related to the time and to the metal enrichment, while the strength of the spiral shock wave probably does not change much with time. 
We express the relation in the form $\mathrm{d} M_{\star} / d t \propto\left(M_{\text {gas }}\right)^{k}$. To demonstrate the nature of the problem, we give the analytical solution for $k=1$. We assume instantaneous recycling. i.e. a fraction $r$ of the gas going into stars. $d M_{*}$, is returned immediately to the interstellar medium. Hence, the total mass, $M_{\text {tot }}=(1-r) M_{*}+M_{\text {gas }}$. We find, (for solutions of this as well as the general case, see Audouze et al, 1975):

$$
\begin{aligned}
M_{\text {gas }} / M_{\text {tot }} & =\exp (-t / \tau) \\
\mathrm{d} M_{\star} / d t & =M_{\text {gas }} / \tau(1-r)
\end{aligned}
$$

Thus, given $M_{\text {gas }} / M_{\text {tot }}$ at the present time $t=1.410^{10} \mathrm{yr}$, we may calculate $\tau$, the rate constant and then predict the present value of $\mathrm{d} M_{\star} / \mathrm{dt}$. This is to be compared with the observed value. MS made this comparison and found reasonable agreement between the $k=1$ analytical solution and the observed rates averaged over annuli in the Galaxy. They assumed a Salpeter (1955) initial luminosity function and the corresponding stellar values from Mezger et al (1974).

Both 'observed' and predicted star formation rates depend on the adopted IMF. The IMF is commonly derived from star counts in the solar neighbourhood. In order to relate the function for stars $>2 \mathrm{M}_{\odot}$ (with ages much less than the age of the Galaxy) to that for low mass stars, one makes an assumption about the rate of star formation over the lifetime of the Galaxy. Salpeter assumed a constant rate of star formation and arrived at an IMF that is well represented by a power law with an exponent -1.35. However, if one allows for some dependence on the amount of gas available (or any other function that decreases with time), the derived IMF for stars $<0.8 \mathrm{M}_{\odot}$ is greatly reduced. Fig. 11 shows the derived curves for a rate dependent on $\left(M_{g a s}\right)^{k}$ where $k$ is $O$ (the salpeter function) $0.5,1$ or 2 . (Curves of this type were first derived and discussed by Schmidt (1963, but see also 1959)).

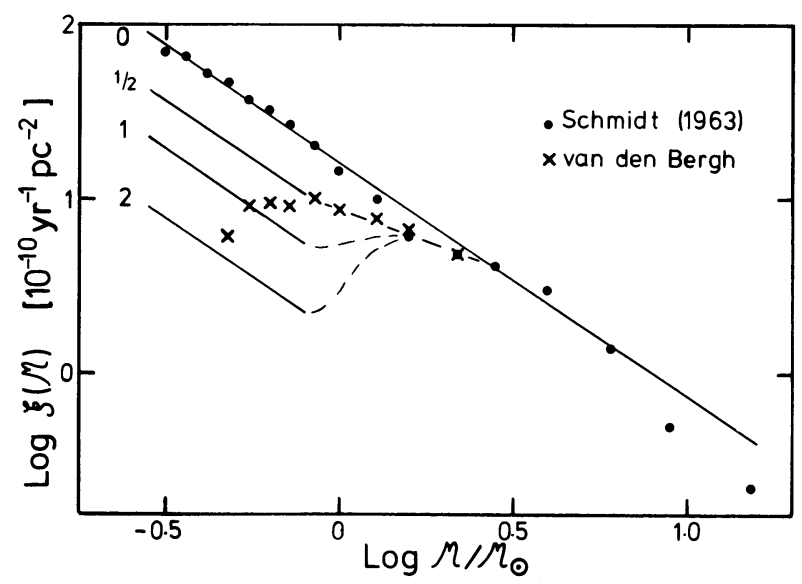

Figure 11: The initial mass function (IMF) derived from star counts in the solar neighbourhood for a star formation rate proportional to $\left(M_{\text {gas }}\right)^{k}$. The dots are from star counts and the $\mathrm{M}_{\mathrm{V}}-\mathrm{M}$ relation tabulated by Schmidt (1963) and an age of the Galaxy of $1.410^{10}$ years. Crosses are van den Bergh's (1961) cluster function, converted to an IMF with the same $M_{V}-M$ scale. 
Thus, one must be selfconsistent and use an IMF that corresponds to the same value of $\mathrm{k}$ for both. Increasing $\mathrm{k}$ has three consequences: 1. The mass of stars associated with a given number of photons decreases, so the observed star formation rate decreases.

2. The 'return rate' is higher, so the predicted star formation rate increases.

3. The rate of star formation in the past relative to the present is increased - which causes the predicted present rates to decrease.

The results are sensitive to the cut-offs of the IMF. The results presented here are for an IMF truncated at 0.1 and $85 \mathrm{M}_{\odot}$ the lower cut off may be unrealistically large.

Fig. 12 shows the 'observed' rates for $k=1 / 2$. The conversion factor from photon rate is $1.175\langle M\rangle /\left\langle N_{C}\right\rangle t(H I I)$, where $\langle M\rangle /\left\langle N_{C}\right\rangle$ is the mass of stars per Lyman continuum photon $=3.410^{-47} \mathrm{M}_{\odot} /$ photon $\sec ^{-1} .^{*}$ )

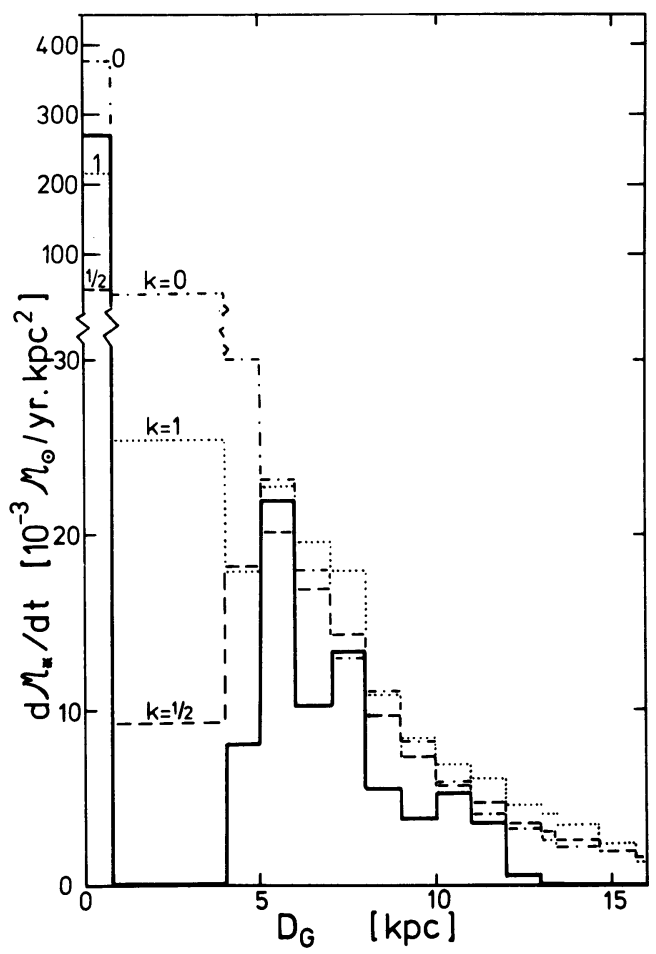

Figure 12: Observed star formation rates averaged over annuli in the galactic plane, compared to predicted rates for various values of $\mathrm{k}$. The values on the ordinate are correct for $k=1 / 2$; for $\mathrm{k}=0,1,2$ they must be multiplied by $1.49,0.74,0.58$, respectively.

*) This differs from the value from Mezger et al (1974 because of the different IMF, different mass limits (Mezger et al used 35 and $0.6 \mathrm{M}_{0}$ ) and because Mezger et al made an error of $1 \mathrm{n} 10$; that the numbers difer by only $30 \%$ is coincidence. As a result, SBM's derived star formation rates are not significantly different from MS's. 
$t$ (HII) is the lifetime of radio HII regions, estimated by SBM at $510^{5}$ years. 1.175 is the correction factor for non-giant HII regions in interarm regions. The entire factor equals $810^{-53} \mathrm{M}_{\circ}$ year $^{-1} /$ photon $\mathrm{sec}^{-1} \mathrm{ab}-$ sorbed in giant HII regions. For a given value of $k$, the possible error is $(+100 \%,-25 \%)$ in $\langle M\rangle /\left\langle\mathrm{N}_{C}\right\rangle$ due to uncertainty in the mass limits; the difference between various $\mathrm{k}^{\prime} \mathrm{s}$ is $250 \%$. Uncertainty in $\mathrm{t}$ (HII) is a factor of 2 and thus the uncertainty in the conversion factor is large. However, the shape of the star formation rate as a function of distance from the center depends only on the accuracy of the photon counting (which is high) and the assumption that the conversion factor is constant throughout the Galaxy. The latter is probably a reasonable assumption in all spiral arm regions, but is ad hoc for the galactic center.

The predicted star formation rates for various values of $\mathrm{k}$ were derived from numerical models of the type described by Biermann (1976). stars are evolved according to tracks in the modern literature and, at the end of the red giant phase, stars between 0.7 and $6.7 \mathrm{M}_{\odot}$ return all but $0.7 \mathrm{M}_{\odot}$ to the IS medium and stars $>6.7 \mathrm{M}_{\odot}$ return all but $1.4 \mathrm{M}_{\odot}$. The fraction $\odot$ f material so returned, averaged over the IMF, is the 'return fraction', $x$. The returned material has heavy element abundances as calculated by Talbot and Arnett (1974) and zero deuterium.

\begin{tabular}{|c|c|c|}
\hline$k$ & $z$ & $z_{D}$ \\
\hline 2 & 0.16 & $10^{-9}$ \\
\hline 1 & 0.10 & $1.310^{-6}$ \\
\hline $1 / 2$ & 0.06 & $1.510^{-5}$ \\
\hline obs. & 0.02 & $2.010^{-5}$ \\
\hline
\end{tabular}

Table 1

Properties of Models

For $\mathrm{D}_{\mathrm{G}}>5 \mathrm{Kpc}$, the relationship between observed and predicted rates does not depend significantly on $\mathrm{k}$. Only for $\mathrm{D}_{\mathrm{G}}<5 \mathrm{Kpc}$, where the remaining gas fraction is very small, does $k$ become critical. Of the models calculated, $k=1 / 2$ gives the best fit, showing a minimum in the 1-4 $\mathrm{kpc}$ range, as observed.

SBM also predict other parameters of the Galaxy, of which the $D$ and heavy element abundances are extremely sensitive to k. Predicted values for the present time in the solar neighbourhood are given in Table 1 for $k=1 / 2,1$ and 2. The sensitivity of $z$ and $z_{D}$ to $k$ is due to the corresponding change in the IMF, which, for $k>0$ has many more massive stars. Thus, $k=1$ or 2 produce far too many metals, by factors of 8 \& 5 respectively. $k=1 / 2$ is best, producing only a factor 3 too many metals. This is probably within the accuracy of Talbot and Arnett's enrichment calculations.

Deuterium is destroyed by astration: the fraction of material astrated during the life of the Galaxy depends on $k$, We adopt an initial value of the $\mathrm{D}$ abundance equal to $10^{-4}$ by mass, near to the maximum allowed by 
Wagoner's (1971) models and the observed present density of galaxies in the universe $\left(\rho_{G}=210^{-31} \mathrm{~g} \mathrm{~cm}^{-3}\right)$. The observed present value (Rogerson and York, 1973) is (1.4 \pm 0.2$) 10^{-5}$ by number. Of the models calculated, $\mathrm{k}=2$ predicts destruction of essentially all primordial $\mathrm{D}, \mathrm{k}=1$ leaves a value 15 times less than observed, $k=1 / 2$ leaves a value only a factor 1.3 less than observed.

$k=1 / 2$ would appear to be the solution providing fewest problems, from the point of view of: aesthetics, i.e. a monotonic IMF (see Schmidt 1963 and Fig. 11); agreement with the cluster IMF (see Fig. 11); prediction of $\mathrm{dM}_{*} / \mathrm{dt}$ as a function of $\mathrm{D}_{\mathrm{G}}$; and prediction of local metal and $\mathrm{D}$ abundances. Audouze et al (1975) get reasonable agreement for isotope ratios with $k=1$ and $r=0.5$. If an IMF extending to $0.01 M_{\odot}$ were adopted in the present experiment, the derived $k$ would increase to $\sim 1$.

$k=1 / 2$ to 1 is a lower dependence of the gas mass than is generally favoured. We emphasize that, in this experiment, the mass of gas is a parameter for time, metal abundance, etc. Our conclusion is that $\mathrm{d} M_{\star} / \mathrm{dt}$, as a function of time, in our Galaxy, is best represented as a dependence on $\left(M_{\text {gas }}\right)^{1 / 2}$.

3) As a Function of Distance from the Galactic Center

The SBM analysis considers evolution of each annulus of the Galaxy, assumed isolated from all others, as a function of time. The rate constant, $\tau$, for each annulus, is set to obtain the present day ratio of gas to stellar mass. We plot, in Fig. 13, Q, the inverse of the proportionality constant between $d M_{*} / d t$ and $\left(M_{\text {gas }}\right)^{k}$ i.e.

$$
\begin{gathered}
d M_{\star} / d t=Q^{-1}\left(M_{\text {gas }}\right)^{k} \\
\text { where } Q=\tau(1-r)\left(M_{\text {tot }}\right)^{k-1}
\end{gathered}
$$

No matter what $\mathrm{k}$ one adopts, $\mathrm{Q}$ increases with distance from the center. This presumably corresponds, physically, to decreasing strength of the shock waves on the inner edges of the spiral arms.

Fig. 13 also shows $z$ and $y$ predicted from the model with $k=1 / 2$, and observed $y^{+}$and $\mathrm{T}_{e}$ for galactic HII regions. The predicted $\mathrm{Z}$ is normalized to 0.02 in the solar neighbourhood. $y$ assumes a primodial value of $7 \%$ and is normalised to $10 \%$ in the solar neighbourhood, i.e. $y=7+1.5 z$ where $y$ is a number fraction and $\mathrm{z}$ is a mass fraction, according to common usage. The observed points, both $y^{+}$and $T_{e}$, are from Churchwell et al (in preparation). The decrease in $\mathrm{T}_{e}$ from 10 to $5 \mathrm{kpc}$ (first noted by Churchwell and Walmsley, 1975 and confirmed by Viner et al, 1976) can only be explained by an increase in $z$ by a factor 2. This would logically be associated with a corresponding increase in the dust/gas ratio. If the dust is selectively absorbing (as asserted by Mezger et al, 1974; Mezger \& Smith, 1976) it will account for the change in $y^{+}$over this radius interval. $\mathrm{T}_{\mathrm{e}}$ does not, however, decrease further from 5 to $\mathrm{O} \mathrm{Kpc}$, indica- 


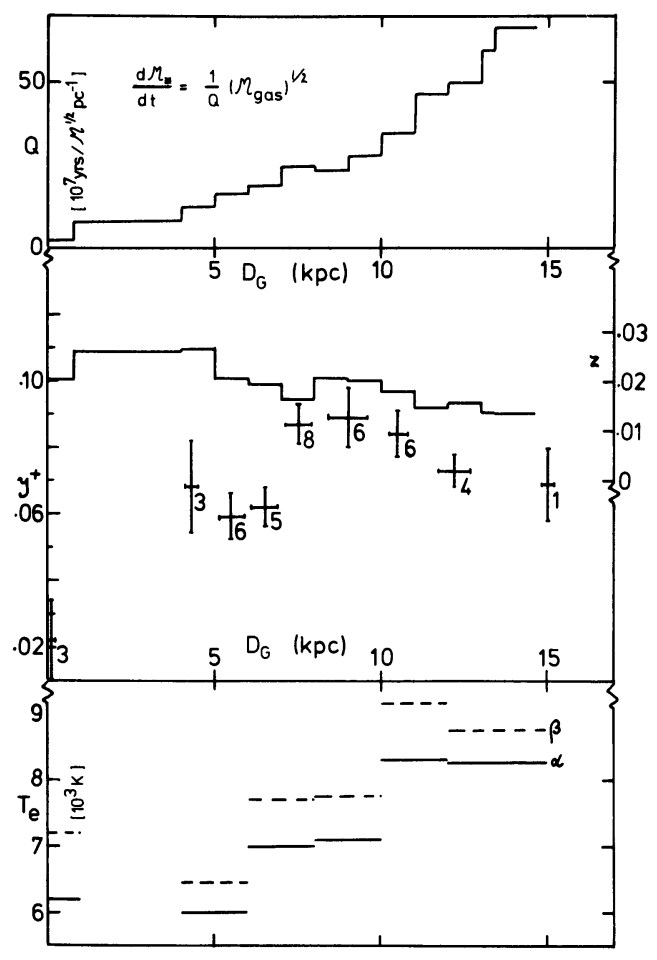

Figure 13: Observed and predicted $(k=1 / 2)$ properties of the Galaxy as a function of distance from the center: a) the inverse of the proportionality constant; b) predicted $\mathrm{Z}$ and $y$ compared to observed $y^{+}$ vertical error bars are mean errors, the number of HII regions in each interval is indicated; c) median electron temperatures of HII regions derived from $\alpha$ and $\beta$ line to continuum ratios. Observed data is from Churchwell et al (in preparation).

ting no further rise in $\mathrm{z}$, which is in agreement with the model predictions. The reason for the flat $\mathrm{Z}$ distribution and high star formation rate at low $D_{G}$ is the return of material from large numbers of low mass stars.

Returning to the star formation rates: The agreement between observed and predicted rates in the main spiral arm region, between 5 and $12 \mathrm{Kpc}$ is good. Outside $12 \mathrm{Kpc}$, the observed rate is about three orders of magnitude too low. There are several possible explanations, of which the most likely is that radial flow has reduced $M_{\text {gas }} / M_{\text {tot }}$ from the value, $\sim 1$, corresponding to the low star formation rate observed. An alternative explanation is that the star formation rate has been underestimated. However, the observed $7 \% \mathrm{He}^{+}$abundance in the outer region supports a low star formation rate. The low $\mathrm{He}^{+}$abundance is derived from 5 HII regions at $\mathrm{D}_{\mathrm{G}}>12 \mathrm{Kpc}$; it cannot be explained by ionization effects. The value agrees with that derived by d'Odorico et al (1976) based on two planetary nebulae at $\mathrm{D}_{\mathrm{G}}>12 \mathrm{Kpc}$, and probably represents the primordial He abundance, there having been essentially no star formation and no enrichment in these regions.

The region between 0.8 and $4 \mathrm{Kpc}$ is more complex. M gas is low and $M_{\text {gas }} / M_{\text {tot }}$ even lower. For a high return rate, it is difficult to achieve a very low gas fraction in a closed volume. It is probable that the 0 star formation rate is less than expected because there are no shock waves to trigger their formation. The gas fraction is probably kept small by a high formation rate of low mass stars and/or mass flow into the galactic center. 
In the center itself, $\mathrm{k}=1 / 2$ predictes $5310^{-10} \mathrm{M}_{\odot} \mathrm{yr}^{-1} \mathrm{pc}^{-2}$ compared to $27010^{-10}$ observed. The predicted rate in this region is enormously sensitive to $\mathrm{k}$. It will also be increased if there is gas flow from the surrounding area. Thus, it appears plausible that the very high rate in the galactic center can be maintained by returned mass from stars and inflow from the immediate surrounding region of the disk. It is also possible that the star formation rate is not constant and/or that the IMF in this region has more massive stars.

We want to thank all our colleagues from the MPIfR and from abroad whose communication of not yet published results and whose critical remarks helped us to prepare this review paper.

\section{REFERENCES}

Audouze, J., Lequeux, J., Vigroux, L. 1975, Astron. Astrophys. 43, 71. Beetz,. M., Elsässer, H., Weinberger, R. 1974, Astron. Astrophys. 34, 335. Beetz, M., Elsässer, H., Poulakos, C., Weinberger, R. 1976, Astron. Astrophys. $50,41$.

Biermann, P. 1976, Astron. Astrophys. (in press).

Blaauw, A., 1964, Ann. Rev. Astron. Astrophys. 2, 213.

Blair, G.N., Peters, W.L., Vanden Bout, P. 1975, Astrophys. J. 200, L161.

Brown, R.L., Gammon, R.H., Knapp, G.R., Balick, B. 1974, Astrophys. J. $192,607$.

Brown, R.L., Zuckerman, B. 1975, Astrophys. J. 202, L125.

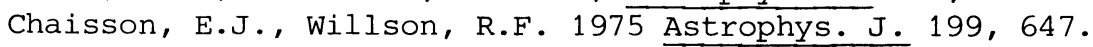

Churchwell, E., Walmsley, C.M. 1975, Astron. Astrophys. 38, 451.

Davidson, K., Harwit, M. 1967, Astrophys. J. 148, 443.

Davies, R.D. 1974, IAU Symposium No. 60, ed. F.J. Kerr and S.C. Simonson

(Reidel, Dordrecht), p. 275.

Dickman, R.L. 1975a, Unpublished thesis, Columbia University.

Dickman, R.L. 1975b, Astrophys. J. 202, 50.

Downes, D. 1974, Proc. Of the ESLAB Symposium HII Regions and the Galactic Center, ESRO-SP105, ed. A.F.M. Moorwood, p.247.

Elmegreem, B.G., Lada, Ch.J. 1976, (preprint).

Emerson, J.P., Furniss, I., Jennings, R.E. 1975, Mon. Not. R. Astr. Soc. $172,411$.

Emerson, D.T. 1974, Mon. Not. R. Astr. Soc. 169, 607.

Encrenaz, P.J., Falgarone, E., Lucas, R. 1975, Astron. Astrophys. 44, 73.

Fazio, G.G., Wright, E.L., Zeilik II, M., Low, F.J. 1976, Astrophys. J. 206, L165.

Gatley, I., Becklin, E.E., Mathews, K., Neugebauer, G., Penston, M.V., Scoville, N. 1974, Astrophys. J. 191, L121.

Georgelin, Y.M., Georgelin, Y.P. 1976, Astron. Astrophys. 49, 57.

Gordon, M.A., Burton, W.B. 1976, Astrophys. J. 208, 346.

Grasdalen, G.L., Strom, K.M., Strom, S.E. 1973, Astrophys. J. 184, L53.

Grasdalen, G.L. 1976, Astrophys. J. 205, L83.

Habing, H.J., Goss, W.M., Matthews, H.E., Winnberg, A. 1974, Astron. Astrophys. 35, 1.

Harris, S., Wynn-Williams, C.G. 1976 Mon. Not. R. Astr. Soc. 174, 649.

Harris, S., Scott, P.F. 1976, Mon. Not. R. Astr. Soc. 175, 371. 
Harten, R. H. 1976, Astron. Astrophys. 46, 109

Harvey, P. M., Gatley, I., Werner, M. W., Elias, J. H., Evans II, N. J., Zuckerman, B., Morris, G., Sato, T., Litvak, M. M. 1974, Astrophys. J. 189, L87

Herbig, G. H. 1960, Astrophys. J. Suppl. 4, 337

Herbig, G. H. 1962, Adv. Astron. Astrophys. 7, 47

Herbig, G. H. 1970, Proc. of the XVIth Liège Symp. 59, 13

Herbst, E., Klemperer, W. 1976, Phys. Today 29, 32

Hudson, H. S., Soifer, B. T. 197 $\overline{6}$, Astrophys. J. 206, 100

Hughes, V. A., Viner, M. R. 1976, Astrophys. J. 204, 55

Iben, I., Talbot, R. J. 1966, Astrophys. J. 144, 968

Israel, F. P., Habing, H. J., de Jong, T. 1973, Astron. Astrophys. 27, 143

Israel, F. P. 1976, Astron. Astrophys. 48, 193

Kahn, F. D. 1974, Astron. Astrophys. 37, 149

Knapp, G. R., Brown, R. L., Kuiper, T. B. H. 1975, Astrophys. J. 196, 167

Krügel, E., Mezger, P. G. 1975, Astron. Astrophys. 42,441

Kutner, M. L., Evans II, N. J., Tucker, K. D. 1976 (preprint)

Lada, C. J., Gull, T. R., Gottlieb, C. A., Gottlieb, E. W. 1976a,

Astrophys. J. 203, 159

Lada, C. J., Dickinson, D. F., Gottlieb, C. A., Wright, E. L. 1976b,

Astrophys. J. 207, 113

Lada, Ch. J. 1976, Astrophys. J. Suppl. Series (in press)

Larson, R. B. 1969a, Mon. Not. R. Astr. Soc. 145, 271; 1969b, ibid 145, 297

Larson, R. B., Starrfield, S. 1971, Astron. Astrophys. 13, 190

Lect. Notes in Phys. 42, 1975, Proc. Of the Symposium HII regions and re-

lated topics, eds. T. L. Wilson and D. Downes (Springer Verlag, Heidelberg)

Liszt, H. S. 1973, Unpubl. thesis, Princeton Univ.

Lo, K. Y. 1974, Unpubl. thesis, Mass. Inst. Technology.

Lo, K. Y., Burke, B. F., Haschick, A. D. 1975a, Astrophys. J. 202, 81

Lo, K. Y., Walker, R. C., Burke, B. F., Moran, J. M., Johnston, K. J.,

Ewing, M. S. 1975b, Astrophys. J. 202, 650

Loren, R. B., Vanden Bout, P.A., Davis, H. J. 1973, Astrophys. J. 185, L67

Loren, R. B. 1975, Nat. Sci. Foundation Technical Rep. MPS-05070-75-1

Mader, G. L., Johnston, K. J., Moran, J. M., Knowles, S. H., Mango, S. A., Schwartz, P. R., Waltman, W. 1975, Astrophys. J. 200, L111

Madore, B. F., Van den Bergh, S., Rogstad, D. H. 1974, Astrophys. J. 191, 317

Mathews, W. 1969, Astrophys. J. 157, 583

Mathewson, D. S., Kruit, P. C. van der, Brouw, W. N. 1972, Astron. Astrophys. 17,468

Mezger, P. G. 1971, Highlights of Astronomy 2, ed. C. de Jager (Reidel, Dordrecht) p. 367

Mezger, P. G., Smith, L. F., Churchwell, E. 1974, Astron. Astrophys. 32, 26y

Mezger, P. G., Wink, J. 1974, Mem. Soc. Astr. Italiana, 45, 315

Mezger, P. G. 1974, PrOC. ESO/SRC/CERN Conference of Research Programmes for the New Large. Telescopes, ed. A. Reiz, p. 79

Mezger, P. G. 1975, Structure and Evolution of Galaxies, ed. G. Setti, (Reidel, Dordrecht) p. 174

Mezger, P. G., Smith, L. F. 1975, Stars and Galaxies from observational points of view, ed. E. K. Kharadze, (Acad. Sci. Georgian SSR) p. 369

Mezger, P. G., Wink, J. 1976, Astrophys. Space Sci. Library (Reidel, Dordrecht) in press 
Mezger, P. G., Smith, L. F. 1976, Astron.Astrophys. 47, 143

Milman, A. S. 1975, Unpubl. thesis, Univ. of Maryland

Milman, A. S., Knapp, G. R., Kerr, F. J., Knapp, S. L., Wilson, W. J. 1975, Astrophys. J. 80, 93

d'odorico, S., Peimbert, M., Sebbadin, F. 1976, Astron. Astrophys. 47, 341

Pankonin, V., Walmsley, C. M. 1976, Astron. Astrophys. 48, 341

Penzias, A. 1975, Atomic and Molecular Physics and the Interstellar

Matter, ed. Roger Balian, (North-Holland, Amsterdam), p. 375

Roberts, M. S. 1957, Publ. Astr. Soc. Pacific 69, 59

Rogerson, J. B., York, D. G. 1973, Astrophys. J. 186, L95

Salpeter, E. E. 1955, Astrophys. J. 121, 161

Schmidt, M. 1959, Astrophys. J. 129, 243

Schmidt, M. 1963, Astrophys. J. 137, 758

Scoville, N. Z., Solomon, P. M., Penzias, A. A. 1975, Astrophys. J. 201,352

Simon, M., Righini, G., Joyce, R. R., Gezari, D. Y 1973. Astrophys. J. 186, L127

Snyder, L. E., Buhl, D. 1975, Astrophys. J. 197, 329

Strel'nitskii 1975, Sov. Phys. - Usp. 17, 507

Sullivan III, W. T., Downes, D. 1973, Astron. Astrophys. 29, 369

Talbot, R. J. Jr., Arnett, W. D. 1974, Astrophys. J. 190, 605

Van den Bergh, S. 1961, Astrophys. J. 134, 554

Viner, M. R., Clarke, J. N., Hughes, V. A. 1976, Astrophys. J.( in press)

Vrba, F. J., Strom, K. M., Strom, S. E., Grasdalen, G. L. 1975, Astrophys. J. 197, 77

Wagoner, R. V. 1971, Highlights of Astronomy 2, ed. C. de Jager, (Reidel, Dordrecht), p. 301

Williams, I. P., Cremin, A. W. 1969, Mon. Not. R. Astr. Soc. 144, 359

Woodward, P. R. 1976, Astrophys. J. 207, 484

Wynn-Williams, C. G., Becklin, E. E., Neugebauer, G. 1972, Mon. Not. R. Astr. Soc. 160, 1

Wynn-Williams, C. G., Becklin, E. E. 1974, Publ. Astr. Soc. Pacific 86, 5

Yorke, H. W., Krügel, E. 1976, Astron. Astrophys. (in press) 